\title{
Simulation of the pressure field beneath a turbulent boundary layer using realizations of uncorrelated wall plane waves
}

5

10

\author{
Laurent Maxit \\ Univ Lyon, INSA-Lyon, LVA EA677 \\ Laboratoire Vibrations- Acoustique \\ Bât. St. Exupéry, 25 bis av. Jean Capelle \\ F-69621 Villeurbanne Cedex, France \\ laurent.maxit@insa-lyon.fr
}

\begin{abstract}
This paper investigates the modelling of a vibrating structure excited by a turbulent boundary layer (TBL). Although the wall pressure field (WPF) of the TBL constitute a random excitation, the element-based methods generally used for describing complex mechanical structures consider deterministic loads. The response of the structure to a random excitation like TBL is generally deduced from calculations of numerous Frequency Response Functions. The result is that the process requires costly computational resources. To tackle this issue, an efficient process is proposed for generating realizations of the WPF corresponding to the TBL. This process is based on a formulation of the problem in the wave-number space and the interpretation of the wall pressure field as uncorrelated wall plane waves. Once the WPF have been synthesized, the local vibroacoustic responses are calculated for the different realizations and averaged together in the last step. A numerical application of this process to a plate beneath a TBL is used to verify its efficiency and ability to reproduce the partial space correlation of the excitation. Finally an application on a stiffened panel modelled with the finite element method is proposed to illustrate the interest of the proposed process.
\end{abstract}

15

Running title: Numerical synthesis of random pressure field

Keywords: Synthesis, random pressure field, turbulent boundary layer, vibroacoustic response 


\section{INTRODUCTION}

Structures excited by the turbulent boundary layer (TBL) are very common in practical applications. Cars, airplanes, trains and submarines may be excited by pressure fluctuations due to the turbulent flow caused by their movements. In order to reduce the noise radiated from or transmitted by these structures, it is important to understand at the design stage how the structure reacts to TBL excitation. It is then necessary to develop numerical tools to predict the vibration or the pressure radiated from the complex panels excited by the turbulent flow. This topic has been the purpose of many works in the literature and it remains an important research topic. To be convince, the reader can find a recent state of the art on this topic in the book [1] published after the FLINOVIA symposium held in 2013 at Roma.

Usually, the calculation process is decomposed into 3 steps:

- First, a hydrodynamic model is used to estimate the TBL parameters (convective velocity, boundary layer thickness, wall shear stress, etc.) over the surface of the structure on the basis of its geometry and the flow conditions.

- Second, the spectrum of the wall pressure fluctuations is evaluated from the TBL parameters estimated previously and by using one of the models proposed in the literature. Some of them are expressed in the space - frequency domain (like the well-known Corcos model [2]), whereas others are in the wavenumber - frequency domain (like the equally wellknown Chase [3] and Smolyakov [4] models). Discussions on different models and comparisons with experiments can be found in [5-8] for the Auto Spectrum Density (ASD) function and in [8-10] for the normalized Cross Spectrum Density (CSD) function.

- Finally, a vibro-acoustic model is used to estimate the response of the structure to the pressure fluctuations.

Many studies were carried out in the past to develop this type of calculation process for predicting the vibro-acoustic response of structures excited by fully developped TBL. The former ones concerned generally simple plates excited by a turbulent flow. In the end of the $60^{\text {th }}$, Strawderman [11] gave a review of existing models (at this period) of finite and infinite plates under turbulence. Although neither the finite nor infinite model agrees wholly with the experimental results, he indicated that the vibration statistics computed from the finite plate model are in better agreement with the experimental results than those computed from the 
infinite panel model. He also investigated with Christman [12] the effect of heavy fluid loading on the vibratory response. Davis [13] proposed a space integration method including the light fluid loading effect to estimate the power density functions of the displacement of the finite plate and of the radiated acoustic pressure. Graham [14] reviewed statistical models of the boundary layer and investigated the more specific case of aircraft statistical models of TBL. The response of a finite panel under boundary layer excitation was determined by using the modal superposition method and a wavenumber integration technique. For naval applications, Ko and Schloemer $[15,16]$ proposed a method for evaluating the transmitted flow noise received by a rectangular hydrophone embedded in an infinite extended viscoelastic layer. The wavenumber filtering effects of both the elastomer layer and the rectangular hydrophone were highlighted by their approach. Mazzoni [17] proposed a deterministic model to approximate the response of an elastic rectangular plate at a low Mach number. The approximation was based on the observations of numerical studies showing that the subconvective region of the turbulent excitation power spectrum contributes significantly to the response of the panel. Rumerman [18-20] derived different expressions giving broad band estimations of the acoustic power radiated from a ribbed plate excited by TBL. He assumed a wavenumber-white pressure excitation and that the ribs radiated independently, that leads the formulations to be more accurate in the high frequency domain. Recently, Ciappi and al. [21] studied numerically and experimentally the response of two composite panels under TBL excitation for nearly subsonic flow conditions. They use literature empirical models for the WPF with the input date obtained from the analyses of experimental wall pressure data. The comparison between finite element and experimental results showed a good agreement between the different results.

For the prediction of the vibratory response of complex panels under TBL excitation, the 90 element-based methods considering deterministic harmonic excitations are generally considered for describing the vibro-acoustic behavior of the panel. Finite Element Modeling (FEM) can be used for a pure structural problem whereas FEM coupled with Perfectly Matched Layers (PMLs) [22], the Boundary Element Method (BEM) [23], or the Infinite Element Model (IEM) [24] can be used for an acoustic radiation problem. The coupling between the statistical model used to describe the wall pressure fluctuations and the deterministic vibroacoustic model represent a difficulty in the calculation process described above. Generally, this coupling is established thanks to a formulation of the random excitation problem in the frequency-space domain [25]. The ASD function of the system response (i.e. structure acceleration, acoustic pressure) at a receiving point is then linked to the CSD function of the wall pressure fluctuations 
through Frequency Response Functions (FRFs) [24]. These FRFs are defined between the receiving point and a set of points distributed on the excited surface. In order to correctly describe the partial correlation of the excitation, it is necessary to consider a large number of points on the excited surface and compute a large number of FRFs [24-25]. A study can be found in [26] which highlights the issues induced by the transformation of the pressure distribution into discrete locations (i.e. nodes), in particular the aliasing effect. Hong and Shin [25] examined in details the maximum mesh size required for reliable finite element analysis. They showed that the mesh size should be defined under consideration of the spatial distribution of the CSD function of the WPF in addition to the dynamic characteristic of the considered structure. This may lead to consider a very fine finite element mesh and it results that the finite element calculations are both time and memory consuming.

Different alternatives have been proposed for overcoming these drawbacks. Ichchou et al. [27] were developed an equivalent "rain on the roof" excitation model for the high frequency range which largely simplified the Finite Element calculations. Hong and Shin [25] proposed

115 an uncorrelated loading model of the WPF which was based on the compensation of the wall pressure correlation lost due to the coarse mesh. A good accuracy with an exact solution was obtained with this approach on a simply supported beam. The proposed loading model can also be applied on more complex structures. In the same time, De Rosa and Franco [28] proposed a scaling procedure in order to reduce the computation cost which can be induced by a high modal density. It consists to reduce the dimensions not involved in the energy transmission whereas the damping is increased in order to keep the same dissipated energy. The same authors and others [29] have also analyzed scaling laws from experimental data involving four plates in air or in water flow. More recently, the same team [30] proposed a frequency modulated pseudoequivalent deterministic excitation. This approach named $\mathrm{PEDE}_{\mathrm{M}}$ is derived from the pseudoexcitation method [31-32]. This latter involves a modal decomposition of the load matrix related to the CSD function of the WPF and it converges to the exact response if all the eigensolutions are considered. Different $\mathrm{PEDE}_{\mathrm{M}}$ approximations were studied for overcoming the drawback of the modal decomposition of the load matrix at each frequency step. The approximations depended on the considered frequency ranges (i.e. low, mid or high) which could be identified with a general criterion given by the authors. They were validated on a chain of linear oscillator and on a flexural plate. Another type of approaches [24, 33] which has been developed recently consists in synthetizing realizations of the wall pressure fluctuations corresponding to the CSD function in the frequency-space domain. The process is based on a Choslesky decomposition 
of the wall pressure CSD matrix [34]. Once the pressure fields corresponding to the different

135 realizations have been obtained, the vibroacoustic model is used to calculate the system response at the receiving point for each realization, separately. The response to the TBL excitation is finally deduced by an ensemble average on the different realizations. With this process, the number of load cases considered in the vibroacoustic calculations corresponds to the number of realizations, which is generally much lower that the number of FRFs considered

140 with the standard approach described above. This is the advantage provided by this approach. However, it always requires a fine finite element mesh of the panel for frequencies above the hydrodynamic coincidence frequency and the Choslesky decomposition is time consuming. In order to tackle these issues, this paper proposes an alternative process to the ones described in $[24,33]$. It is based on a formulation of the random excitation problem in the frequencywavenumber domain and the interpretation of the wall pressure field as uncorrelated wall plane waves. Realizations of the WPF are directly generated from an analytical expression depending on the wavenumber-frequency spectrum of the WPF. The Cholesky decomposition is then not be required. For each realization, the panel response induced by the deterministic WPF (of the considered realization) can then be estimated from a low-frequency deterministic vibroacoustic model of the panel. For instance, it can be achieved by using a finite element model when dealing with a complex panel. The stochastic response of the panel is then obtained from an ensemble average of the different panel responses. The interest of this type of approach is that it requires a relatively small number of realizations for estimating the stochastic response of the panel. This point will be studied on the basic case of a simply supported plate. Moreover,

155 we will highlight how in some situations, the well-known filtering effect of the panel $[35,36]$ can be considered in the process in order to reduce the mesh size of the panel. The accuracy of the proposed process will be studied in function of the WPF model (i.e. Corcos or Chase), the convective velocity of the flow and the panel thickness. A stiffened plate modelled with the finite element solver MSC/NASTRAN will also be considered to illustrate the interest of the present approach for practical application.

The paper is organized as follows:

- Section 2 gives a description of the problem considered and presents the outlines of its mathematical formulation in the frequency-wavenumber space;

- Section 3 introduces the concept of uncorrelated wall plane waves and proposes the process for synthetizing realizations of the WPF; 
- The numerical process for estimating the panel response to TBL excitation is summarized in Sec. 4.

- Then, in Sec. 5, numerical applications are proposed for a basic case of studying the influence of different parameters on the accuracy of the approach proposed.

- Finally, before the concluding remarks, an application on a stiffened plate is proposed in Sec. 6.

\section{VIBRATING PANELS EXCITED BY RANDOM}

\section{A. Presentation of the problem}

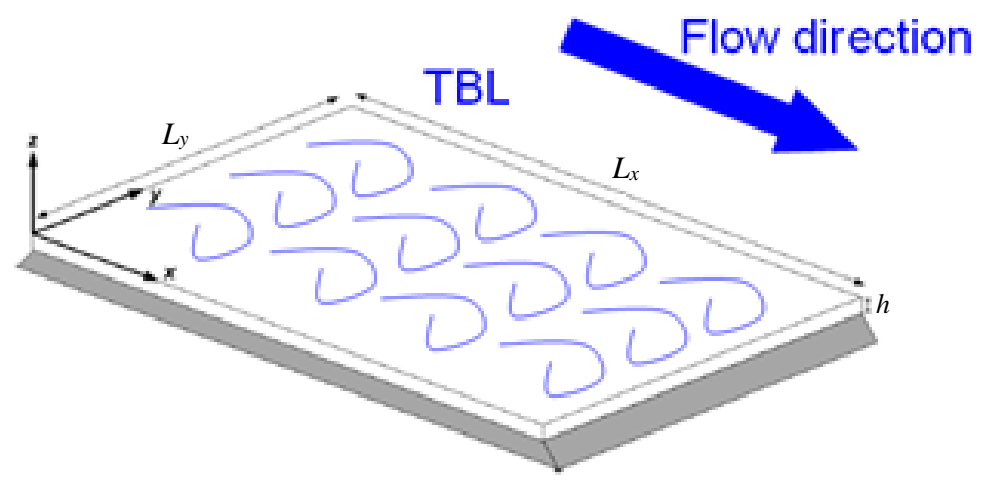

Fig. 1. Baffled simply supported plate excited by a homogeneous and stationary TBL.

Let us consider a baffle panel of surface $\Sigma_{p}$ excited by a TBL as shown in Fig. 1. We assume that the TBL is fully developed, stationary, and homogeneous over $\Sigma_{p}$. Moreover, the panel and the boundary layer are assumed to be weakly coupled. It is then assumed that the vibration of the plate does not interfere with the wall pressure. The spectrum of the wall pressure fluctuations over a rigid surface can then be considered for charactering the panel excitation. This can be estimated from the parameters characterizing the turbulent boundary layer (i.e. convective velocity, $U_{c}$; boundary layer thickness; wall shear stress), and one of the wall pressure models proposed in the literature [2-7]. The space-frequency cross spectrum of the 
wall pressure fluctuations $S_{p p}^{T B L}\left(\mathbf{x}-\mathbf{x}^{\prime}, \omega\right)$ may be written on the specific form proposed by Graham [10] and reused by different authors ([5, 37]):

$$
S_{p p}^{T B L}\left(\mathbf{x}-\mathbf{x}^{\prime}, \omega\right)=S_{p p}(\omega)\left(\frac{U_{c}}{\omega}\right)^{2} \bar{S}_{p p}^{T B L}\left(\mathbf{x}-\mathbf{x}^{\prime}, \omega\right),
$$

where:

- $S_{p p}(\omega)$ (in $\mathrm{Pa}^{2} / \mathrm{Hz}$ ) is the Auto Spectrum Density (ASD) function of the WPF depending on the angular frequency $\omega$, and;

- $\bar{S}_{p p}^{T B L}\left(\mathbf{x}-\mathbf{x}^{\prime}, \omega\right)\left(\right.$ in $\left.\left.\operatorname{rad}^{2} / \mathrm{m}^{2}\right)\right)$ is the normalized Cross Spectrum Density (CSD) function of the WPF depending on the spatial separation between two points, $\mathbf{x}-\mathbf{x}^{\prime}$, and the angular frequency.

This form can be used with different models for the ASD function and for the normalized CSD function, independently one from each other. For example, the Goody [6] or Rozenberg

200 [7] models can be used for the ASD function whereas the Corcos [2] or Chase [3] models can be considered for the normalized CSD functions.

It is assumed that the panel has a linear vibroacoustic behavior that can be represented by a deterministic model (like FEM). It may be complex. That is to say, for example, that it could be made of different layers of different materials. It could also be stiffened by ribs on the side opposite the flow.

The goal for us consists in estimating the vibrations of this panel when it is excited by the wall pressure fluctuations induced by the TBL. In the next section, we give the outlines of the 210 formulation of this problem in the frequency-wavenumber space. Details of the formulation can be found in the literature [38-40].

\section{B. Mathematical formulation}

$p_{b}(\tilde{\mathbf{x}}, \tau)$ represents the wall-pressure fluctuations due to the TBL on the plate at point $\mathbf{x}$ as a

215 function of time $t$. The plate acceleration at point $\mathbf{x}$ due to wall-pressure fluctuations, $\gamma(\mathbf{x}, t)$, can be expressed as the convolution product 


$$
\gamma(\mathbf{x}, t)=\int_{\Sigma_{p}-\infty}^{+\infty} \int_{\gamma} h_{\gamma}(\mathbf{x}, \tilde{\mathbf{x}}, t-\tau) p_{b}(\tilde{\mathbf{x}}, \tau) d \tau d \tilde{\mathbf{x}},
$$

where $h_{\gamma}(\mathbf{x}, \tilde{\mathbf{x}}, t)$ is the acceleration impulse response at point $\mathbf{x}$ for a normal unit force at point $\tilde{\mathbf{x}}$.

As the turbulent flow induces a random process, the plate response is characterized by the auto correlation function of the acceleration, $R_{r \gamma}$. Assuming that the process is stationary and ergodic (i.e. expectation replaced by the limit of a time average), $R_{\gamma \gamma}$ can be written as:

$$
R_{\gamma \gamma}(\mathbf{x}, t)=\lim _{T \rightarrow \infty} \frac{1}{T} \int_{-T / 2}^{T / 2} \gamma(\mathbf{x}, t) \gamma(\mathbf{x}, t+\tau) d \tau .
$$

By introducing (2) in (3) and taking the time Fourier transform of the result, we obtain the Auto Spectrum Density (ASD) of the acceleration at point $\mathbf{x}$ (see details in [38-40]):

$$
S_{\gamma \gamma}(\mathbf{x}, \omega)=\int_{\Sigma_{p} \Sigma_{p}} H_{\gamma}^{*}(\mathbf{x}, \tilde{\mathbf{x}}, \omega) S_{p p}^{T B L}(\tilde{\mathbf{x}}-\tilde{\widetilde{\mathbf{x}}}, \omega) H_{\gamma}(\mathbf{x}, \tilde{\widetilde{\mathbf{x}}}, \omega) d \tilde{\mathbf{x}} d \tilde{\tilde{\mathbf{x}}}
$$

where $H_{\gamma}(\mathbf{x}, \tilde{\mathbf{x}}, \omega)=\int_{-\infty}^{+\infty} h_{\gamma}(\mathbf{x}, \tilde{\mathbf{x}}, t) e^{-j \omega t} d t$ is the Frequency Response Function (FRF) in terms of acceleration at point $\mathbf{x}$ for a normal force at point $\tilde{\mathbf{x}}$ and the asterisk denotes the complex conjugate.

Now, let us consider the space Fourier transform of the wall pressure spectrum, $\phi_{p p}^{T B L}(\mathbf{k}, \omega)$. This is related to the wall pressure spectrum in the physical space $S_{p p}^{T B L}\left(\mathbf{x}-\mathbf{x}^{\prime}, \omega\right)$ by

$$
S_{p p}^{T B L}\left(\mathbf{x}-\mathbf{x}^{\prime}, \omega\right)=\frac{1}{4 \pi^{2}} \int_{\infty} \phi_{p p}^{T B L}(\mathbf{k}, \omega) e^{i \mathbf{k} \cdot\left(\mathbf{x}-\mathbf{x}^{\prime}\right)} d \mathbf{k} .
$$

Introducing Eq. (5) in Eq. (4) gives

$$
S_{\gamma \gamma}(\mathbf{x}, \omega)=\frac{1}{4 \pi^{2}} \int_{\infty} \phi_{p p}^{T B L}(\mathbf{k}, \omega) \tilde{H}_{\gamma}(\mathbf{x}, \mathbf{k}, \omega)^{2} d \mathbf{k},
$$

with

$$
\tilde{H}_{\gamma}(\mathbf{x}, \mathbf{k}, \omega)=\int_{\Sigma_{p}} H_{\gamma}(\mathbf{x}, \tilde{\mathbf{x}}, \omega) e^{j \mathbf{k} \tilde{\mathbf{x}}} d \tilde{\mathbf{x}}
$$


$\tilde{H}_{\gamma}(\mathbf{x}, \mathbf{k}, \omega)$ is generally called the sensitivity function [41]. The interpretation of Eq. (7) indicates that this quantity corresponds to the acceleration at point $\mathbf{x}$ when the panel is excited by a unit wall plane wave of wavevector $\mathbf{k}$ (i.e. by a WPF $p_{b}(\tilde{\mathbf{x}})=e^{j \mathbf{k} \tilde{\mathbf{x}}}, \forall \tilde{\mathbf{x}} \in \Sigma_{p}$ ).

In Eq. (5-6), improper integrals exist over the wavenumber space. In the following, it is assumed that they can be approximated by considering the rectangular rule and by truncating and regularly sampling the wavenumber space. The criterion for defining the cut-off wavenumbers and the wavenumber resolutions will be discussed later. However, it should be underlined here that the cut-off wavenumbers can be different between Eq. (5) related to the wall pressure and Eq. (6) related to the panel vibration due to the well-known filtering effect of the panel [35]. $\Pi_{p}$ and $\Pi_{\gamma}$ denote the sets of wavenumbers selected to estimate Eq. (5) and (6), respectively. Thus the following can be written:

$$
\begin{aligned}
& S_{p p}^{T B L}\left(\mathbf{x}-\mathbf{x}^{\prime}, \omega\right) \approx \frac{1}{4 \pi^{2}} \sum_{\varsigma \in \Pi_{p}} \phi_{p p}^{T B L}\left(\mathbf{k}_{\varsigma}, \omega\right) e^{i \mathbf{k}_{\varsigma}\left(\mathbf{x}-\mathbf{x}^{\prime}\right)} \Delta \mathbf{k}, \\
& S_{\gamma \gamma}(\mathbf{x}, \omega) \approx \frac{1}{4 \pi^{2}} \sum_{\varsigma \in \Pi_{\gamma}} \phi_{p p}^{T B L}\left(\mathbf{k}_{\varsigma}, \omega\right)\left|\tilde{H}_{\gamma}\left(\mathbf{x}, \mathbf{k}_{\varsigma}, \omega\right)\right|^{2} \Delta \mathbf{k},
\end{aligned}
$$

245 where $\Delta \mathbf{k}$ represents the wavenumber resolutions.

The outlines of the formulation for estimating the vibratory response of the panel have been presented here. One can emphasize that these developments can be easily adapted for evaluating the noise radiated by the panel (see the details of the formulation in [38-40]). It is however outside the scope of the present paper which focus on the synthetize of the WPF and the prediction of the panel vibration.

\section{UNCORRELATED WALL PLANE WAVE FIELD}

The basic idea of the proposed approach is to represent the TBL pressure CSD function as the result of a combination of uncorrelated wall pressure plane waves. This approach may be related to room acoustics, where a diffuse field can be represented by summing the effect of an infinite number of acoustic plane waves originating from all spatial directions and having the same amplitude [42].

This section is organized as follow: we define first the concept of uncorrelated wall plane waves. Then, we establish the link between the uncorrelated wall plane waves and the TBL 
excitation. Finally, we propose a process for synthetizing realizations of the WPF induced by uncorrelated wall plane waves. As the uncorrelated wall plane waves can be representative of the TBL excitation when the wave amplitudes are correctly defined, the process which will be described in this section will permit to synthetize realizations of the WPF induced by a TBL excitation.

\section{A. Definition}

Let us define the concept of uncorrelated wall plane waves. One recalls that the term wall plane wave refers to the blocked pressure acting on a panel surface varying spatially as $e^{j \mathbf{k} \mathbf{x}}, \forall \mathbf{x} \in \Sigma_{p}$ where $\mathbf{k}$ is the wavevector of the wave considered. It is assumed that this wave has a stochastic amplitude. The blocked pressure induced by a wall plane wave $\varsigma$ of wavenumber $\mathbf{k}_{\varsigma}$ can then be written

$$
p_{\varsigma}(\mathbf{x}, t)=\operatorname{Re}\left\{A_{\varsigma}(t) e^{j \mathbf{k}_{\varsigma} \mathbf{x}}\right\},
$$

where $A_{\varsigma}(t)$ is a random variable.

275 This wall plane wave is clearly a surface wave in the sense that it is only defined at the surface of the panel. Moreover, we underline that the wavevector $\mathbf{k}$ may be arbitrary in the 2-D real space $\mathbf{k} \in \mathbb{R}^{2}$. It does not depend on the acoustic propagation as it is the case for an acoustic plane wave.

The pressure CSD function corresponding to this wall plane wave is therefore:

$$
S_{p_{\varsigma} p_{\varsigma}}\left(\mathbf{x}-\mathbf{x}^{\prime}, \omega\right)=S_{A_{\varsigma} A_{\zeta}}(\omega) e^{j \mathbf{k}_{\varsigma}\left(\mathbf{x}-\mathbf{x}^{\prime}\right)},
$$

280 where $S_{A_{s} A_{s}}(\omega)$ is the ASD function of the wave amplitude.

Now, let us consider a set of Uncorrelated Wall Plane Waves (UWPW) of wavenumbers $\mathbf{k}_{\varsigma}, \varsigma \in \Pi$. The total blocked pressure $p(\mathbf{x}, t)$ is given by:

$$
p(\mathbf{x}, t)=\sum_{\varsigma \in \Pi} p_{\varsigma}(\mathbf{x}, t) \text {. }
$$

As the wall plane waves are assumed to be uncorrelated, the CSD function between the amplitudes of two different waves is null: $S_{A_{\varsigma} A_{c^{\prime}}}(\omega)=0$ if $\varsigma \neq \varsigma^{\prime}$. Hence the CSD function of the pressure induced by this set of uncorrelated wall plane waves is therefore: 


$$
S_{p p}^{U W P W}\left(\mathbf{x}-\mathbf{x}^{\prime}, \omega\right)=\sum_{\varsigma \in \Pi} S_{A_{\varsigma} A_{\varsigma}}(\omega) e^{j \mathbf{k}_{\varsigma}\left(\mathbf{x}-\mathbf{x}^{\prime}\right)} .
$$

\section{B. Uncorrelated wall plane wave field and TBL excitation}

Now let us establish a link between the UWPW and the TBL excitation. To do that, one considers a set of UWPW defined such that $\Pi=\Pi_{p}$ and the ASD functions of the wave amplitudes are given by:

$$
S_{A_{\varsigma} A_{\varsigma}}(\omega)=\frac{\phi_{p p}^{T B L}\left(\mathbf{k}_{\varsigma}, \omega\right) \Delta \mathbf{k}}{4 \pi^{2}}, \forall \varsigma \in \Pi .
$$

By introducing Eq. (14) in Eq. (13) and by comparing the results with Eq. (8), it can be seen immediately that $S_{p p}^{U W P W}\left(\mathbf{x}-\mathbf{x}^{\prime}, \omega\right) \approx S_{p p}^{T B L}\left(\mathbf{x}-\mathbf{x}^{\prime}, \omega\right)$. This clearly demonstrates that the TBL excitation can be represented as a superposition of uncorrelated wall pressure plane waves.

It is possible to verify that this representation of the TBL excitation remains consistent with the panel response. Indeed, if we consider a set of UWPW with $\Pi=\Pi_{\gamma}$, the ASD function of the panel acceleration in response to the set of UWPW may be written as:

$$
S_{\gamma \gamma}^{U W P W}(\mathbf{x}, \omega)=\sum_{\varsigma^{\prime} \in \Pi_{\gamma}} \sum_{\zeta \in \Pi_{\gamma}} \tilde{H}_{\gamma}\left(\mathbf{x}, \mathbf{k}_{\varsigma}, \omega\right) S_{A_{\varsigma^{\prime}} A_{\xi^{\prime}}}(\omega) \overline{\tilde{H}_{\gamma}\left(\mathbf{x}, \mathbf{k}_{\varsigma^{\prime}}, \omega\right)},
$$

which is simplified because the wall plane waves are uncorrelated:

$$
S_{\gamma \gamma}^{U W P W}(\mathbf{x}, \omega)=\sum_{\varsigma \in \Pi_{\gamma}} S_{A_{\varsigma} A_{\zeta}}(\omega)\left|\tilde{H}_{\gamma}\left(\mathbf{x}, \mathbf{k}_{\varsigma}, \omega\right)\right|^{2} .
$$

300 When defining the wave amplitudes by Eq. (14), the direct comparison of Eq. (16) and Eq. (9) gives $S_{\gamma}^{U W P W}(\mathbf{x}, \omega) \approx S_{\gamma}^{T B L}(\mathbf{x}, \omega)$.

In conclusion, the TBL excitation can be represented by a set of UWPW when the wave amplitudes are defined with Eq. (14).

\section{Realizations of uncorrelated wall plane wave fields}

305 Let us now define a process for synthetizing realizations of the WPF induced by UWPW. A set of UWPW as defined in the previous section constitutes a random excitation. One way of approximating it is to consider different realizations of the random pressure field. Using the physical interpretation of uncorrelated wall plane waves, it is possible to define the wall pressure field of the $k^{\text {th }}$ realization, $p^{k}(\mathbf{x}, \omega)$ by, 


$$
p^{k}(\mathbf{x}, \omega)=\sum_{\zeta \in \Pi} \sqrt{S_{A_{\zeta} A_{\zeta}}(\omega)} e^{j \varphi_{\zeta}^{k}} e^{j \mathbf{k}_{\varsigma} \mathbf{x}}
$$

where $\varphi_{\varsigma}^{k}$, are random phase values uniformly distributed in $[0,2 \pi]$.

In this expression, the terms $e^{j \varphi_{s}^{k}}$ express the fact that the wall plane waves are uncorrelated and the terms $\sqrt{S_{A_{s} A_{s}}(\omega)}$ represent the wave amplitudes. The root square of the ASD function of the wave amplitudes is used to counteract the second-moment related to the ASD function.

It can be easily demonstrated that the CSD function of the wall pressure corresponding to an

315 infinite number of these realizations, $S_{p p}^{S}\left(\mathbf{x}-\mathbf{x}^{\prime}, \omega\right)$, corresponds well to the CSD function of the uncorrelated wall pane wave field, $S_{p p}^{U W P W}\left(\mathbf{x}-\mathbf{x}^{\prime}, \omega\right)$. Indeed, $S_{p p}^{S}\left(\mathbf{x}-\mathbf{x}^{\prime}, \omega\right)$ can be written by definition:

$$
S_{p p}^{S}\left(\mathbf{x}-\mathbf{x}^{\prime}, \omega\right)=E\left[\sum_{\varsigma \in \Pi} \sqrt{S_{A_{\zeta} A_{\zeta}}(\omega)} e^{j \varphi_{\varsigma}^{k}} e^{j \mathbf{k}_{\varsigma} \mathbf{x}} \overline{\sum_{\zeta \in \Pi} \sqrt{S_{A_{\varsigma} A_{s}}(\omega)} e^{j \varphi_{\varsigma}^{k}} e^{j \mathbf{k}_{\varsigma} \mathbf{x}^{\prime}}}\right]_{k},
$$

where $E[]_{k}$ represents the ensemble average over the realizations and the upper bar denotes the complex conjugate of the complex number.

By rearranging the different terms,

$$
S_{p p}^{S}\left(\mathbf{x}-\mathbf{x}^{\prime}, \omega\right)=\sum_{\zeta \in \Pi} \sum_{\xi^{\prime} \in \Pi} \sqrt{S_{A_{s} A_{s}}(\omega) S_{A_{s^{\prime}} A_{s^{\prime}}}(\omega)} e^{j\left(\mathbf{k}_{\xi^{\prime}} \mathbf{x}-\mathbf{k}_{\xi^{\prime}} \mathbf{x}^{\prime}\right)} E\left[e^{j\left(\varphi_{\varphi^{k}}^{k}-\varphi_{\xi^{\prime}}^{k}\right)}\right],
$$

and by considering an infinite number of realizations,

$$
E\left[e^{j\left(\varphi_{s}^{k}-\varphi_{s^{\prime}}^{k}\right)}\right]_{k}= \begin{cases}1, & \text { if } \varsigma=\varsigma^{\prime}, \\ 0, & \text { otherwise },\end{cases}
$$

we obtain $S_{p p}^{S}\left(\mathbf{x}-\mathbf{x}^{\prime}, \omega\right)=S_{p p}^{U W P W}\left(\mathbf{x}-\mathbf{x}^{\prime}, \omega\right)$.

This demonstrates well that when considering an infinite number of realizations, the CSD function of the WPF defined by Eq. (17) is equivalent to the CSD function of the uncorrelated wall pane wave field.

To summarize this section 3, one can emphasize that: (a), the TBL excitation can be 330 represented by a set of uncorrelated wall plane waves when the wave amplitudes are defined by Eq. (14); (b), Realizations of the random pressure field corresponding to a set of uncorrelated wall plane waves can be obtained using Eq. (17); (c), The CSD function of the WPF 
corresponding to these realizations converge to the CSD function of the set of uncorrelated wall plane waves when the number of realization tends to infinity.

It results that realizations of the random pressure field corresponding to a TBL excitation can be obtained by using Eq. (17) when the wave amplitudes are defined by Eq. (14). The CSD function of the WPF corresponding to an infinite number of realizations is then equal to the CSD function of the TBL excitation. An infinite number of realizations of the WPF as described previously are then equivalent to the TBL excitation. In consequence, the response of the panel under TBL excitation can be estimated from the response of the panel excited by the WPF corresponding to these realizations. In practice, a finite number of realizations $K$ will be considered to approximate the TBL excitation. The accuracy of the calculation process summarized in section 4 will be studied in section 5 in function of the number of realizations.

\section{PROCESS FOR ESTIMATING THE PANEL RESPONSE FROM THE WALL PRESSURE REALIZATIONS}

The following is a description of the numerical process for estimating the panel response to a TBL excitation from the realizations of the uncorrelated wall plane wave field. This process is directly derived from the previous section. One has seen that the realizations of the WPF defined by Eq. (17) with Eq. (14) are representative of the WPF of the TBL excitation. The proposed process consist then to estimate the panel response to the WPF of each individual realization and then to average the obtained panel responses over the different realizations.

Let us consider a deterministic vibroacoustic model of the panel in order to estimate the panel response to deterministic load cases. This model can be an analytical model for an academic structure or an element-based model for a complex panel (as it will be illustrated in section 6 for a stiffened plate represented by a finite element model within the MSC/NASTRAN code).

The numerical process proposed can be decomposed into three steps:

- The first step consists in calculating the WPF of $K$ realizations of the uncorrelated wall plane wave field representing the TBL excitation. It is carried out by using the formula (17) and considering the wave amplitudes defined by Eq. (14). When using an element-based method for describing the vibro-acoustic behavior of the panel, the WPF should be applied to the nodes 
of the mesh belonging to the interaction surface between the panel and the flow. For the node $i$ of this set of nodes, the pressure corresponding to the $k^{\text {th }}$ realization (deduced from Eq. (14) and (15)) is given by:

$$
p^{k}\left(x^{i}, y^{i}, \omega\right)=\sum_{\varsigma \in \Pi} \sqrt{\frac{\phi_{p p}^{T B L}\left(k_{x}^{\varsigma}, k_{y}^{\varsigma}, \omega\right) \delta k_{x} \delta k_{y}}{4 \pi^{2}}} e^{j\left(k_{x}^{\zeta} x^{i}+k_{y}^{\zeta} y^{i}+\varphi_{\varsigma}^{k}\right)},
$$

where:

$370-x$ and $y$ represent the axis in the streamwise direction and the crosswise direction, respectively;

$-\left(k_{x}^{\varsigma}, k_{y}^{\varsigma}\right)$ are the coordinates of the wavevector $\mathbf{k}_{\varsigma}$;

$-\left(x^{i}, y^{i}\right)$ are the coordinates of node $i$.

- $\delta k_{x}$ and $\delta k_{y}$ are the wavenumbers resolutions in the spanwise and streamwise directions,

375 respectively, and;

- $\varphi_{\varsigma}^{k}$, are random phase values uniformly distributed in $[0,2 \pi]$;

- In the second step, the vibroacoustic model is used to estimate $\gamma^{k}(\mathbf{x}, \omega)$, the panel acceleration at point $\mathbf{x}$ when the panel is excited by the deterministic WPF, $p^{k}(\mathbf{x}, \omega)$ calculated in the previous step. When an element-based model is considered, expression (21) is directly used to prescribe the pressure on the nodes at the interface between the panel and the flow. A direct frequency analysis can then be performed for example to estimate the panel acceleration $\gamma^{k}$. This calculation is repeated for the different realizations $k \in\{1, \ldots, K\}$. The number of load cases considered in the vibroacoustic simulations therefore corresponds to the number of realizations;

- Finally, in the last step, the ASD of the acceleration at point $\mathbf{x}$ is estimated by an ensemble average of the acceleration responses, $\gamma^{k}(\mathbf{x}, \omega), k \in\{1, \ldots, K\}$ estimated in the previous step:

$$
S_{\gamma \gamma}^{S}(\mathbf{x}, \omega) \approx E\left[\gamma^{k}(\mathbf{x}, \omega) \overline{\gamma^{k}(\mathbf{x}, \omega)}\right]_{k \in\{1, \ldots, K\}}
$$

390

$S_{\gamma \gamma}^{S}(\mathbf{x}, \omega)$ corresponds then to the ASD of the acceleration at point $\mathbf{x}$ induced by the WPF of the $K$ realizations. As the CSD function of the WPF of the $K$ realizations converges to the CSD 
function of the WPF of the TBL excitation when $K \rightarrow+\infty, S_{\gamma \gamma}^{S}(\mathbf{x}, \omega)$ converge to the ASD function of the plate acceleration at point $\mathbf{x}$ induced by the TBL excitation. In practice, a finite number of realizations $K$ will be considered to approximate the ASD function of the plate acceleration. This will be studied in the next section.

\section{NUMERICAL APPLICATIONS}

400 For evaluating the numerical process described in the previous section, we are going to compare its results on a basic application case with the results obtained by a direct calculation of Eq. (9). In the following, the latter calculation is named the sensitivity function method whereas the numerical process described in section 4 is called the sampling method.

A. Presentation of the test case

The test case considered for this numerical application is composed of a rectangular thin plate simply supported along its four edges and excited by a turbulent air flow. This academic plate was chosen because the modal base can be obtained analytically and the vibratory response can be easily interpreted. The plate is made of aluminum and the flow direction is parallel to the longest edges of the plate (i.e. about the x-axis). The effect of the air on the plate vibrations are neglected. The numerical values of the physical parameters considered for this nominal test case are given in Tab. 1. To study the influence of different parameters on the accuracy of the presented approach, one will be led to modify the physical parameters of the nominal case. This will be indicated in the text. For the sake of compactness, one limits however the study to the cases presenting a hydrodynamic coincidence frequency (i.e. frequency for which the flexural wavenumber is equal to the convective wavenumber) lower than the higher frequency of interest (i.e. frequency for which the flexural wavenumber is equal to the convective wavenumber). In particular, one does not study the cases concerning by a frequency band of interest below the hydrodynamic coincidence frequency. For the nominal case, the 420 hydrodynamic coincidence frequency is $86.7 \mathrm{~Hz}$ and the higher frequency of interest has been fixed to $300 \mathrm{~Hz}$. 


\begin{tabular}{|l|l|}
\hline Parameters & Numerical value \\
\hline Panel thickness & $h=3 \mathrm{~mm}$ \\
\hline Panel length in the streamwise direction & $L_{x}=1.5 \mathrm{~m}$ \\
\hline Panel length in the crosswise direction & $L_{y}=0.9 \mathrm{~m}$ \\
\hline Panel Young's modulus & $E=67 \times 10^{9} \mathrm{~Pa}$ \\
\hline Panel Poisson's ratio & $v=0.34$ \\
\hline Panel mass density & $\rho=2700 \mathrm{~kg} / \mathrm{m}^{3}$ \\
\hline Panel damping loss factor & $\eta=0.01$ \\
\hline Receiving point coordinates & $\left(x_{M}, y_{M}\right)=(0.133 \mathrm{~m}, 0.212 \mathrm{~m})$ \\
\hline Convective velocity & $U_{c}=50 \mathrm{~m} / \mathrm{s}$ \\
\hline TBL displacement thickness & $\delta^{*}=5.3 \mathrm{~mm}$ \\
\hline Friction velocity & $u^{*}=2.6 \mathrm{~m} / \mathrm{s}$ \\
\hline
\end{tabular}

Table 1. Physical parameters of the nominal test case.

Two different models of WPF will be considered in order to study the influence of the model on the accuracy of the results. From Eq. (1) and the definition of the space Fourier transform 430 (5), the CSD function of the WPF in the wavenumber space $\phi_{p p}^{T B L}$ can be written:

$$
\phi_{p p}^{T B L}\left(k_{x}, k_{y}, \omega\right)=S_{p p}(\omega)\left(\frac{U_{c}}{\omega}\right)^{2} \bar{\phi}_{p p}^{T B L}\left(k_{x}, k_{y}, \omega\right),
$$

where $\bar{\phi}_{p p}^{T B L}\left(k_{x}, k_{y}, \omega\right)$ is the normalized CSD function of the WPF in the wavenumber space which is a dimensionless quantity.

The Corcos model is first considered because it provides an analytical expression of the CSD function both in the space-frequency domain $\left(\mathbf{x}-\mathbf{x}^{\prime}, \omega\right)$ [2] and in the wavenumber-frequency domain $(\mathbf{k}, \omega)$. The Corcos model is a semi-empirical model. Although the Corcos model is simple and it is frequently used in the literature, it has been pointed out by different authors that it may be deficient to represent accurately the subconvective domain of the WPF [44]. One can also notice that some improvements have been proposed recently to circumvent this issue [45]. 440 We consider however the Corcos model in the following due its simplicity, without any 
consideration on its ability to reproduce fairly the WPF induced by a TBL. The Corcos normalized CSD function of the WPF used in the following depends on the convective velocity given in Tab. 1 for the nominal case. It is given by ([10], [37]):

$$
\bar{\phi}_{p p}^{T B L}\left(k_{x}, k_{y}, \omega\right)=\frac{4 \alpha_{x} \alpha_{y}}{\left[\alpha_{x}{ }^{2}+\left(1-\frac{U_{c} k_{x}}{\omega}\right)^{2}\right]\left[\alpha_{y}{ }^{2}+\left(\frac{U_{c} k_{y}}{\omega}\right)^{2}\right]},
$$

with the Corcos's parameters: $\alpha_{x}=0.11, \alpha_{y}=0.77$.

The second model of WPF considered for the numerical applications is the Chase model [10, 43]. It has been deduced from theoretical developments based on the Poisson equation and it depends on numerous empirical parameters. It is supposed to be more accurate in the low wavenumber region that the empirical models [45]. The Chase normalized CSD function of the WPF is expressed by Eq. (3.18) to (3.20) of Ref. [10]. The empirical constants given after these equations in this reference are also considered in the present paper. This model depends on the TBL displacement thickness, the friction velocity and the convective velocity which are given on Tab. 1 for the nominal case.

For the sake of simplicity, the vibratory responses of the panel are normalized by $S_{p p}(\omega)\left(\frac{U_{c}}{\omega}\right)^{2}$, which makes it independent of the ASD of the WPF. In others words, this normalized response corresponds to the vibratory response of the panel induced by the WPF defined by the normalized CSD function, $\bar{\phi}_{p p}^{T B L}\left(k_{x}, k_{y}, \omega\right)$ (see Eq. (23)).

\section{B. Numerical modelling}

The vibratory response of the plate to a given pressure field, $p_{e}(x, y, \omega)$ can be easily estimated by using the modal expansion method. The modal angular frequency, $\omega_{m, n}$, the modal shape, $\phi_{m, n}$ and the modal mass, $M_{m, n}$, are given for each couple of non-null integers $(m, n)$ by:

$$
\omega_{m, n}=\sqrt{\frac{D}{\rho h}}\left(\frac{m \pi}{L_{x}}\right)^{2}+\left(\frac{n \pi}{L_{y}}\right)^{2}, \phi_{m, n}(x, y)=\sin \left(\frac{m \pi}{L_{x}}\right) \sin \left(\frac{n \pi}{L_{y}}\right), M_{m, n}=\frac{\rho h L_{x} L_{y}}{4} .
$$

where $D$ is the flexural rigidity given by $D=\frac{E h^{3}}{12\left(1-v^{2}\right)}$. 
For the modal expansion, the modes having a frequency in the extended angular frequency band $\left[0,3 / 2 \omega_{\max }\right]$ are considered, where $\omega_{\max }$ represents the highest angular frequency of interest. $\Gamma=\left\{(m, n) \in \mathbb{N}^{*} \times \mathbb{N}^{*} / \omega_{m, n} \in\left[0,3 / 2 \omega_{\max }\right]\right\}$ denotes the set of modal order couples corresponding to these modes.

To estimate the ASD function of the panel acceleration directly with Eq. (9), it is necessary to estimate the sensitivity functions $\tilde{H}_{\gamma}$ at point $\left(x_{M}, y_{M}\right)$ for each couple of wavenumbers $\left(k_{x}, k_{y}\right) \in \Pi_{\gamma}:$

$$
\tilde{H}\left(x_{M}, y_{M}, k_{x}, k_{y}, \omega\right)=\sum_{(m, n) \Gamma \Gamma} \frac{-\omega^{2} \psi_{m, n}\left(k_{x}, k_{y}\right) \phi_{m, n}\left(x_{M}, y_{M}\right)}{M_{m, n}\left(-\omega^{2}-j \omega \omega_{m, n} \eta+\omega_{m, n}^{2}\right)} .
$$

$\psi_{m, n}$ represents the modal force given by

$$
\begin{gathered}
\psi_{m, n}\left(k_{x}, k_{y}\right)=\int_{0}^{L_{x} \int_{y}} e_{0}^{j k_{x} x+j k_{y} y} \phi_{m, n}(x, y)=I_{m}^{x} I_{n}^{y}, \text { where } \\
I_{p}^{\xi}=\left\{\begin{array}{l}
\left(\frac{p \pi}{L_{\xi}}\right) \frac{(-1)^{p} e^{j k_{\xi} L_{x}}-1}{k_{\xi}^{2}-\left(\frac{p \pi}{L_{\xi}}\right)^{2}}, \text { if } k_{\xi} \neq \frac{p \pi}{L_{\xi}}, \\
\frac{1}{2} j L_{\xi}, \text { otherwise. }
\end{array}\right.
\end{gathered}
$$

475

On the other hand, to estimate the ASD function of the panel acceleration with the numerical process described in Section 4 , it is necessary to estimate $\gamma^{k}\left(x_{M}, y_{M}, \omega\right)$, the acceleration response at point $\left(x_{M}, y_{M}\right)$ induced by the wall pressure (17) corresponding to the $k^{\text {th }}$ realization:

$$
\gamma^{k}\left(x_{M}, y_{M}, \omega\right)=\sum_{(m, n) \in \Gamma} \frac{-\omega^{2} F_{m, n}^{k}(\omega) \phi_{m, n}\left(x_{M}, y_{M}\right)}{M_{m, n}\left(-\omega^{2}-j \omega \omega_{m, n} \eta+\omega_{m, n}{ }^{2}\right.},
$$

where the modal forces are given by:

$$
F_{m, n}^{k}=\sum_{\varsigma \in \Pi} \sqrt{S_{A_{\varsigma} A_{\varsigma}}(\omega)} e^{j \varphi_{\varsigma}^{k}} \psi_{m, n}\left(k_{x}, k_{y}\right) .
$$

The latter expression is well adapted for the present case for which we can calculate an analytical expression of $\psi_{m, n}$ (i.e. Eq. (27-28)). For a complex panel, the forced response or the mode shapes can be calculated by FEM and can be known at discrete points (i.e. the nodes of 
the mesh). In the literature ([24-25], [28]), it has already been shown that the size mesh should be defined carefully for describing correctly the spatial variations of the WPF and for avoiding aliasing phenomenon [25]. In order to study the influence of this type of approximation in the framework of the proposed approach, we also perform an approximation of the modal forces by considering a spatial discretization of the mode shapes by $S$ points along the streamwise direction and $R$ points along the crosswise direction. The modal forces for the $k^{\text {th }}$ realization can then be approximated by using the rectangular rule:

$$
F_{m, n}^{k} \approx \sum_{r=0}^{R} \sum_{s=0}^{S} p^{k}(r \Delta x, s \Delta y, \omega) \phi_{m, n}(r \Delta x, s \Delta y) \Delta x \Delta y
$$

490 with $\Delta x=\frac{L_{x}}{R}, \Delta y=\frac{L_{y}}{S}$.

The spatial resolutions $\Delta x$ and $\Delta y$ can be defined using a criterion based on the TBL characteristics or on the plate characteristics. This point will be studied in section V.D.4.

\section{Definition of the sets of wavenumbers}

Before evaluating the sampling method, one defines the sets of wavenumbers which intervene Eq. (8) and (9) for the sensitivity method and in Eq. (17) and (21) for the sampling method. They were introduced as the results of the truncation and the sampling of the wavenumber space in order to approximate the integrals of Eq. (5) and (6).

The criterion for defining the cut-off wavenumbers in the streamwise and the crosswise directions should be defined such that the significant contributions of the integrands of these equations are taken into account well.

\section{- Definition of the cut-off wavenumber in the streamwise direction}

To highlight the different contributions in the wavenumber space for the nominal test case, we plotted on Fig. 2 the two quantities which intervene in the integrands of Eq. (5) and (6). They are expressed as a function of the streamwise wavenumber $k_{x}$, and the frequency $f$ $(\omega=2 \pi f)$, in the case of the crosswise wavenumber $k_{y}$, fixed at 0 . 
Fig. 2a shows the normalized CSD function of the wall pressure spectrum of the Corcos model. It exhibits the highest values for wavenumbers close to the convective wavenumber $k_{c}$. Furthermore, in Fig. 2b, the highest values of the sensitivity functions (calculated with Eq. (26)) can be observed for frequency and wavenumbers close to the modal frequencies and the modal wavenumbers in the streamwise direction, respectively. To illustrate this, we have indicated the modal frequencies and the modal wavenumbers of the first 10 modes of the plate in Tab. 2. The frequency and wavenumber of the highest amplitudes in Fig. $2 \mathrm{~b}$ correspond well to the modal frequency $f_{m, n}$ and the modal wavenumbers $k_{m}$ of the plate modes with $n=1$. It should be emphasized that only these particular modes have the most significant contributions in the sensitivity functions shown in fig. $2 \mathrm{~b}$ because the crosswise wavenumber is equal to 0 for this figure (i.e. $k_{y}=0$ ). Whatever the case, it can be however concluded that the highest values of the sensitivity functions can be observed for wavenumbers below or close the natural flexural wavenumber of the plate $k_{f}=\sqrt{\omega \sqrt{\rho h / D}}$ (see Fig 2b).
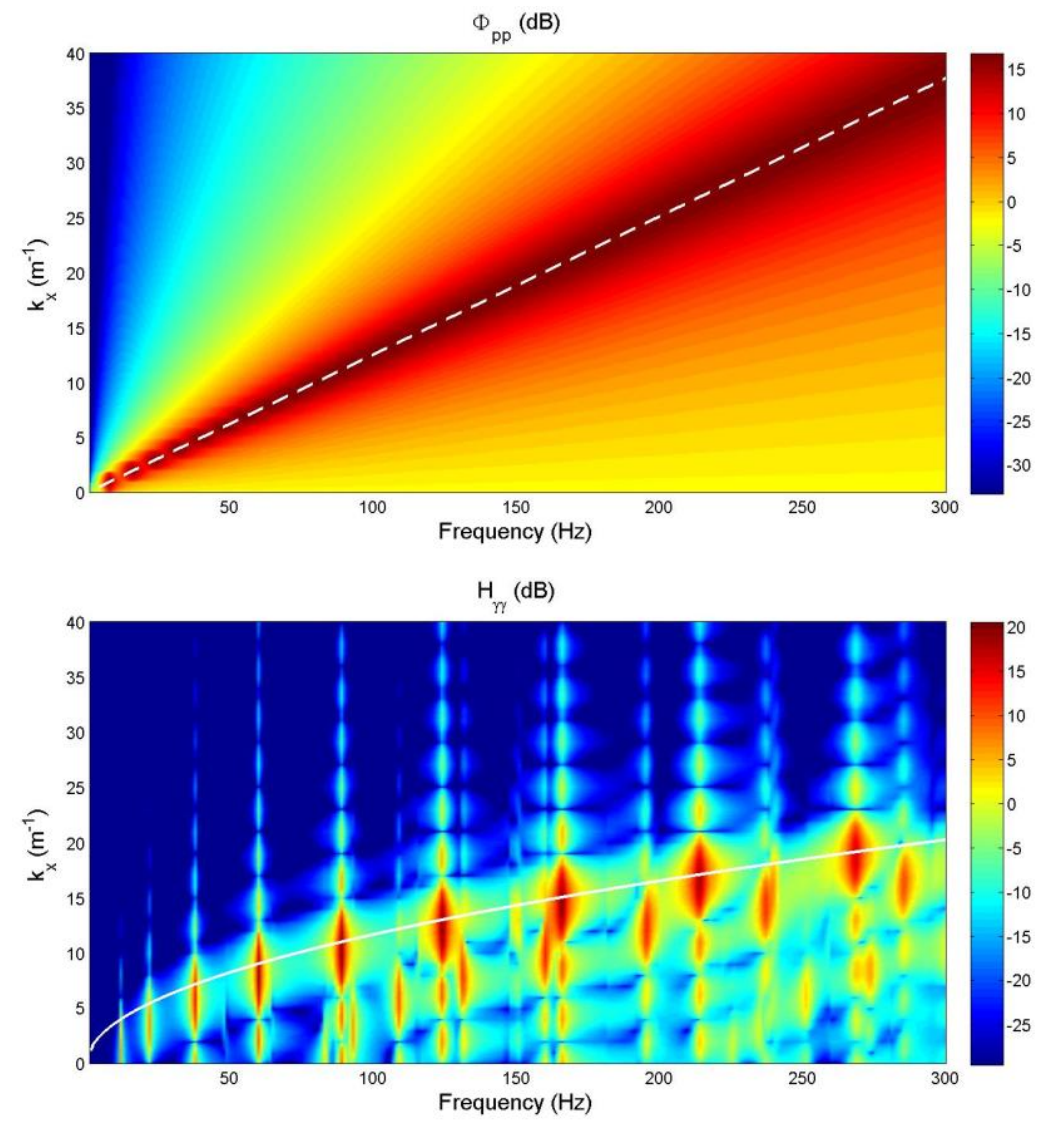

(b)

Fig. 2. (a), The normalized CSD function of wall pressure spectrum given by the Corcos model, $\bar{\phi}_{p p}^{T B L}\left(k_{x}, 0, \omega\right)\left(\mathrm{dB}\right.$, ref. 1); (b), The sensitivity function of the plate at point $\left(x_{M}, y_{M}\right)$,

$$
\mid \bar{H}_{\gamma}\left(x_{M}, y_{M}, k_{x}, 0, \omega\right)^{2}(\mathrm{~dB}, \text { ref. } 1) .
$$




\begin{tabular}{|c|c|c|c|c|}
\hline$f_{m, n}(\mathrm{~Hz})$ & $m$ & $k_{m}=\frac{m \pi}{L_{x}}\left(\mathrm{~m}^{-1}\right)$ & $n$ & $k_{n}=\frac{n \pi}{L_{y}}\left(\mathrm{~m}^{-1}\right)$ \\
\hline 12.1 & 1 & 2.09 & 1 & 3.49 \\
\hline 21.7 & 2 & 4.18 & 1 & 3.49 \\
\hline 37.7 & 3 & 6.28 & 1 & 3.49 \\
\hline 38.7 & 1 & 2.09 & 2 & 6.98 \\
\hline 48.4 & 2 & 4.18 & 2 & 6.98 \\
\hline 60.1 & 4 & 8.37 & 1 & 3.49 \\
\hline 64.4 & 3 & 6.28 & 2 & 6.98 \\
\hline 83.2 & 1 & 2.09 & 3 & 10.47 \\
\hline 86.8 & 4 & 8.37 & 2 & 6.98 \\
\hline 88.9 & 5 & 10.47 & 1 & 3.49 \\
\hline
\end{tabular}

Table 2. Modal information for the first ten modes of the plate: $f_{m, n}$, the modal frequency $\left(\omega_{m, n}=2 \pi f_{m, n}\right) ; m, n$, the modal orders in $x$ and $y$ directions, respectively; $k_{m}, k_{n}$, the modal wavenumbers in the $x$ and $y$ directions.

For defining the set of wavenumbers $\Pi_{p}$ related to the wall pressure field (i.e. Eq. (8)), the cut-off wavenumbers should be defined only from the characteristics of the wall pressure spectrum (as the blocked pressures are independent of the panel). The truncation of the wavenumber space should include the convective ridge in the streamwise direction. Then, one defines the cut-off wavenumber by:

$$
\overline{k_{x}^{p}}=\kappa k_{c}^{\max },
$$

where $k_{c}^{\max }$ is the convective wavenumber at the higher frequency of interest and $\kappa$ is a margin coefficient greater than one. In the following, $\kappa=1.2$ will be considered.

For defining the set of wavenumbers $\Pi_{\gamma}$ related to the panel acceleration, the truncation of the wavenumber space should be done by considering, both, the excitation characteristics and the panel characteristics. Fig. 3a shows the result of the product between and the wall pressure spectrum (i.e. Fig 2a) and the sensitivity function (i.e. Fig 2 b). It should be underlined that this product appears in the summation of Eq. (9) to evaluate the ASD function of the plate 
acceleration. It can be observed on Fig. 3a that the contribution of the convective domain is negligible. This is due to the well-known filtering effect of the pressure fluctuations by the panel $[35,36]$. Thus, for this case, the truncation of the wavenumber space in the streamwise direction can be achieved without considering the convective ridge. The cut-off wavenumber in the streamwise direction used to define the set of wavenumbers $\Pi_{\gamma}$ in Eq. (9) or in Eq. (17) can then be given by:

$$
\overline{k_{x}^{\gamma 1}}=\kappa k_{f}^{\max },
$$

where $k_{f}^{\max }$ is the convective wavenumber at the higher frequency of interest.
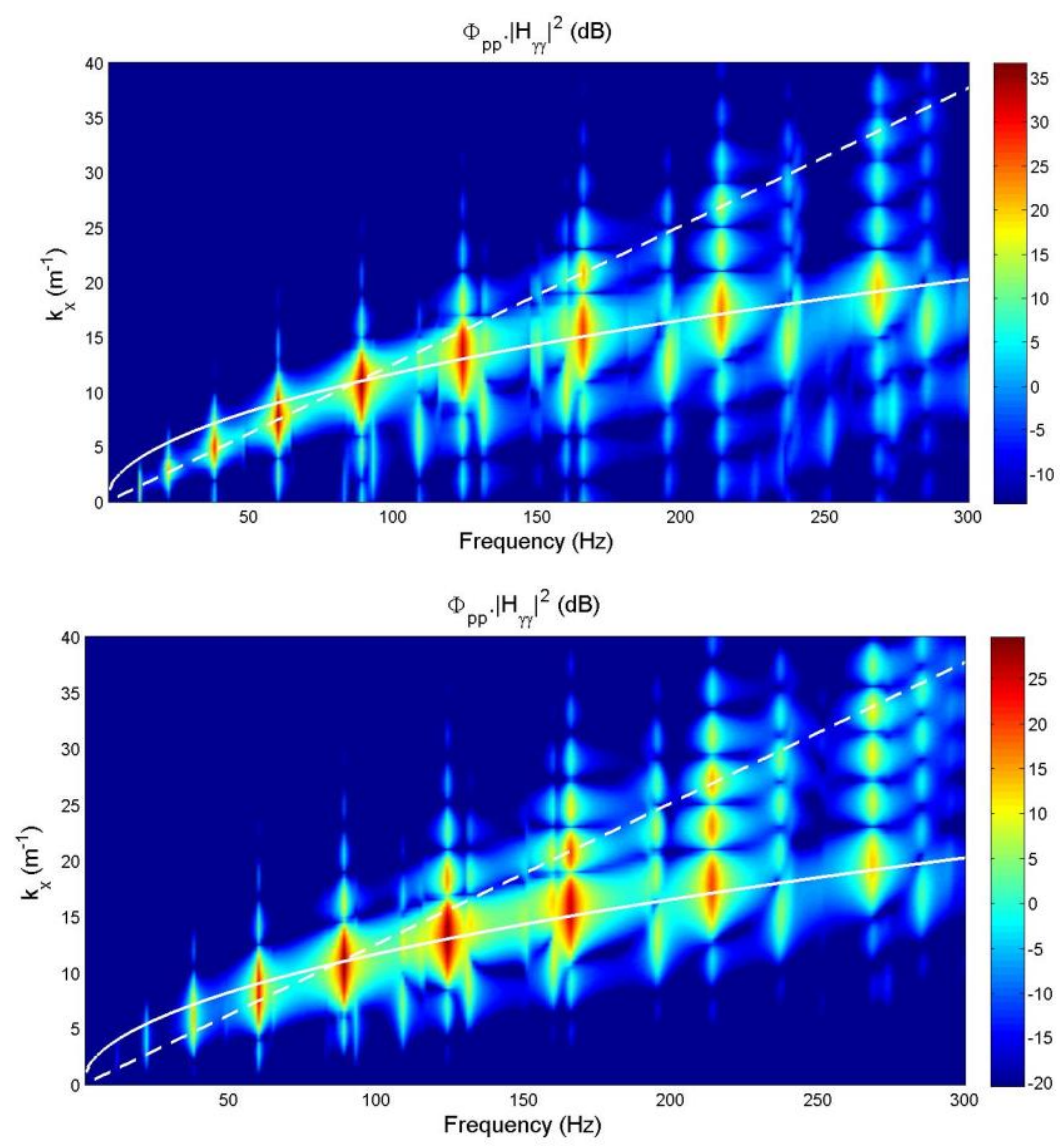

(b)

Fig. 3. Values of the product between the sensitivity function and the wall pressure spectrum, $\bar{\phi}_{p p}^{\text {TBL }}\left(k_{x}, 0, \omega\right)\left|\bar{H}_{\gamma}\left(x_{M}, y_{M}, k_{x}, 0, \omega\right)\right|^{2}$ (i.e. integrand of Eq. (6), dB, ref. 1). Results for two models of wall pressure spectrum: (a), Corcos model; (b), Chase model. Dashed line: convective wavenumber; Solid line: flexural wavenumber.

It should however be mentioned that it is not a general result. The filtering effect of the structure is not always enough important to vanish the contributions of the convective ridge. It 
depends in particular on the frequencies of interest [35], the panel boundary conditions [36], and the considered model of the WPF. This latter dependency is highlighted on Fig. 3b showing the same type of results than Fig. 3a when considering the Chase model. One can observe that the contributions of wavenumbers above the flexural wavenumber are more important with the Chase model than for the Corcos model. This is due to a stronger decrease of the CSD function in the low wavenumber domain of the Chase model compared the Corcos model. For the considered case, the criterion defined by Eq. (33) may be in its limit of validity with the Chase model. This will be verified in the section 5.D.1 In the case of the filtering effect of the structure is not dominant, the criterion (34) based on the TBL characteristics should be applied to estimate the panel response:

$$
\overline{k_{x}^{\gamma 2}}=\overline{k_{x}^{p}}=\kappa k_{c}^{\max } .
$$

\section{- Definition of the cut-off wavenumber in the crosswise direction}

For the crosswise direction, it can be observed in general that the wall pressure spectrum decreases monotonically when the crosswise wavenumber $k_{y}$ increases. The cut-off wavenumber $\overline{k_{y}^{p}}$ related to the wall pressure field (i.e. Eq. (8)) has been fixed at $300 \mathrm{rad} / \mathrm{m}^{-1}$ with a trial-and-error process. For the panel response, the result of the product between the sensitivity functions and the wall pressure spectrum is dominated by the wavenumbers below or close to the natural flexural wavenumber of the plate (results not plotted here). The cut-off wavenumber in the crosswise direction can then be defined as the one in the streamwise direction:

$$
\overline{k_{y}^{\gamma}}=\overline{\kappa k_{f}^{\max }} .
$$

\section{- Wavenumber resolutions}

The wavenumber resolutions in the two directions should be defined such that they correctly represent the spatial variations in the wavenumber space of the wall pressure spectrum and the sensitivity function. The analytical expression of the sensitivity functions for the panel considered (i.e. Eq. (26-28)) gives an order of magnitude of these spatial variations (inversely proportional to the panel lengths) whereas the wall pressure spectrum varies relatively slowly as a function of the wavenumbers. In the following, the wavenumber resolutions are then fixed at $0.25 \mathrm{rad} / \mathrm{m}$, independently of the frequency. For a more complex panel, a trial and error process can be used to fix these parameters. 


\section{Analysis of results}

\section{The sensitivity method}

595 The ASD function of the panel acceleration at the receiving point $M$ has been evaluated with the sensitivity method for the nominal test case. Calculations have been performed for the two cut-off wavenumber criterions (33) and (34) (i.e. $\overline{k_{x}^{\gamma 1}}=24.3 \mathrm{rad} / \mathrm{m}$ and $\overline{k_{x}^{\gamma 2}}=45.2 \mathrm{rad} / \mathrm{m}$, respectively) and for the two models of WPF described in section 5.A (i.e. Corcos and Chase models). The results are plotted on Fig. 4 in function of the frequency. For the Corcos model, a

600 very good agreement between the two calculations are observed on the whole frequency band of interest. This confirms that the structure filters sufficiently the convective ridge of WPF in order that this latter can be neglected. For the Chase model, the agreement between the two calculations is very good up to around $200 \mathrm{~Hz}$. Above this frequency, the calculation considering the criterion (33) underestimates slightly the panel response. A difference of 2.5

$605 \mathrm{~dB}$ can be observed at $300 \mathrm{~Hz}$. This can be explained from the observations made on Fig. $3 \mathrm{~b}$ in the previous section. These results highlights well that the criterion (33) should be used with carefully. We reach its limit of validity for the present case with the Chase model. However for the present case, the prediction remains globally a correct estimation of the plate response.

610 In the following, the results of the sensitivity method using the criterion (34) will be used as a point of comparison in order to evaluate the accuracy of the sampling method. 


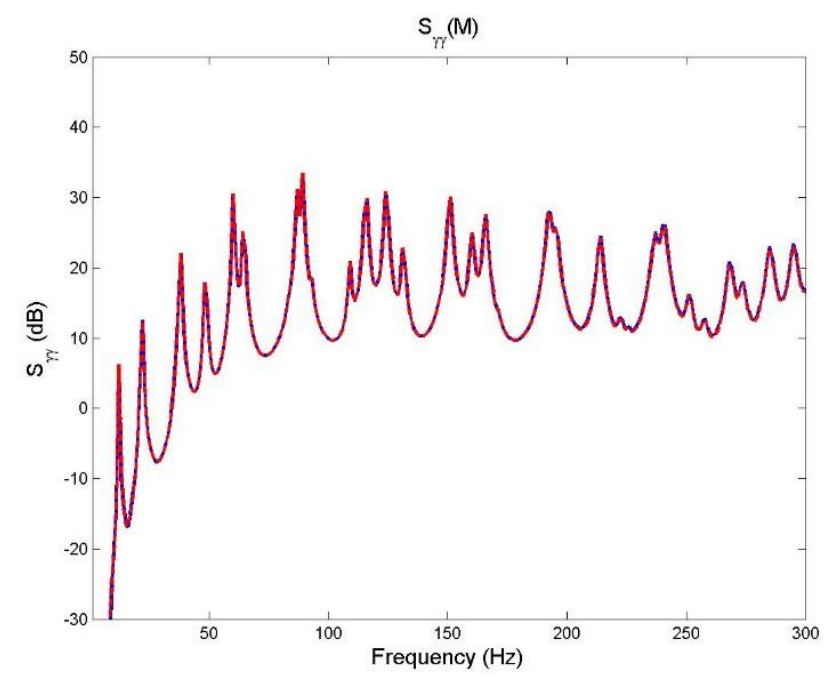

(a)

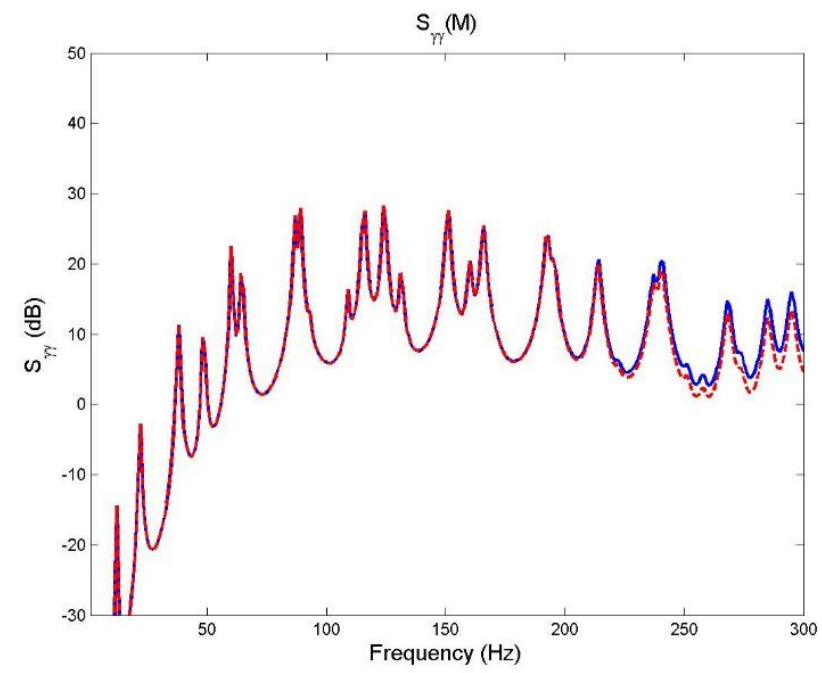

(b)

Fig. 4. ASD function of the panel acceleration at the receiving point $M$ as a function of the frequency, $10 \log _{10}\left[S_{\gamma \gamma}(M)\right]\left(\mathrm{dB}\right.$, ref. $\left.1 \mathrm{~m} / \mathrm{s}^{2} / \mathrm{Hz}^{0.5}\right)$. Comparison of results obtained with the sensitivity function method with different criterion of the truncation of the wavenumber space: Full line, criterion (34) based on the TBL characteristics; dash line, criterion (33) based on the plate characteristics. Results for two models of wall pressure spectrum: (a), Corcos model; (b), Chase model.

\section{Synthesis of the wall pressure field}

The realizations of the wall pressure field are achieved using Eq. (17). By way of illustration, the WPF of two realizations at $300 \mathrm{~Hz}$ are given in Fig. 5 considering the Corcos model:

- The first one (Fig. 5a) has been obtained when the wavenumber set $\Pi$ is defined with the 625 TBL characteristics (i.e. $\Pi=\Pi_{p}$ ). Spatial variations due to wave propagations in the 
streamwise direction appear. The wavelength of these waves is around $0.2 \mathrm{~m}$, which correspond roughly to the convective wavelength $\left(\lambda_{c}=2 \pi / k_{c}=0.16 \mathrm{rad} / \mathrm{m}\right.$ at $\left.300 \mathrm{~Hz}\right)$.

- The second one (Fig. 5b) considers the wavenumber set defined from the panel characteristics $\left(\Pi=\Pi_{\gamma}\right)$. In particular, the criterion (33) is applied to define the cut-off

630 wavenumber in the streamwise direction. One can notice that the spatial variations present higher wavelengths and the amplitudes are lower than in Fig. 5a. This is directly due to the truncations of the wavenumber space which is more restrictive when considering the panel characteristics than the TBL characteristics. The WPF of Fig. 5b does not represent the convective ridge of the TBL. It explains why the amplitudes are lower. However, it represents the part of the pressure field induced by the TBL that contributes to the panel vibration.
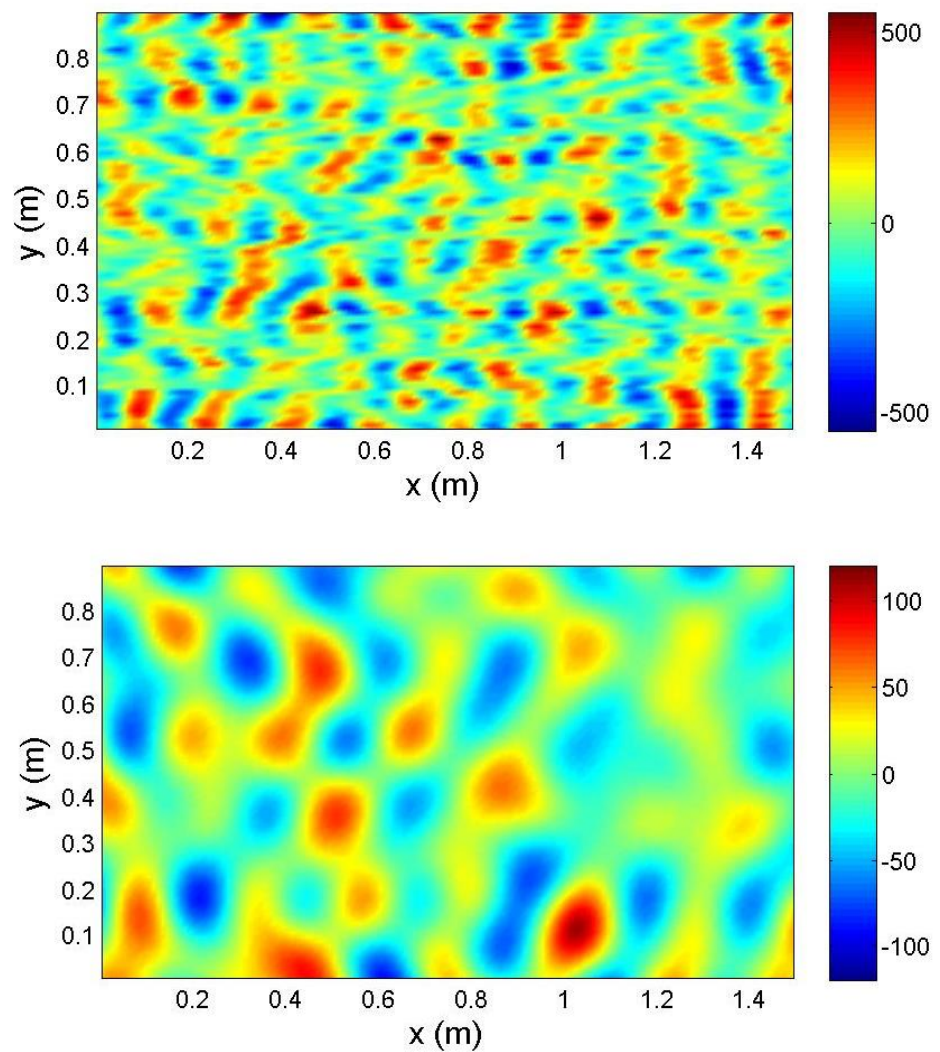

Fig. 5. Two realizations of the WPF at $300 \mathrm{~Hz}$ obtained with Eq. (17): (a), using the wavenumber set defined with the TBL characteristics (i.e. $\left.\Pi=\Pi_{p}\right)$; (b), using the wavenumber set defined with the plate characteristics (i.e. $\Pi=\Pi_{\gamma}$ ).

In order to study the WPF synthetized with Eq. (17), one will compare the spatial coherence estimated from the WPF of $K$ realizations with the one given by the analytical expression of the 
Corcos model. The spatial coherence between point $\mathbf{x}$ and $\mathbf{x}^{\prime}$ can be estimated from $K$ realizations by:

$$
\Gamma\left(\mathbf{x}, \mathbf{x}^{\prime}, \omega\right)=\frac{\left|E\left[p^{k}(\mathbf{x}, \omega) \overline{p^{k}\left(\mathbf{x}^{\prime}, \omega\right)}\right]_{k \in\{1, \ldots, K\}}\right|}{\sqrt{\left.E\left[\mid p^{k}(\mathbf{x}, \omega)\right)^{2}\right]_{k \in\{1, \ldots, K\}} E\left[\left|p^{k}\left(\mathbf{x}^{\prime}, \omega\right)\right|^{2}\right]_{k \in\{1, \ldots, K\}}}} .
$$

645 where $p^{k}(\mathbf{x}, \omega)$ is obtained with Eq. (17).

The results of this equation for $K=30$ and $K=900$ are plotted in Fig. 6 for the streamwise and crosswise directions. It is clear that the small coherence length in the crosswise direction compared to the streamwise direction is well represented by the stochastic process even if only 30 realizations are considered. It can also be seen that a large number of realizations should be considered to correctly represent the small coherences corresponding to a relatively large separation. This seems to indicate that a relatively large number of realizations should be necessary to represent the wall pressure fluctuations finely. However, as the panel filters the wall pressure fluctuations, it is not evident that a large number of realizations remain necessary to evaluate the panel response. This will be the subject in the next sections.
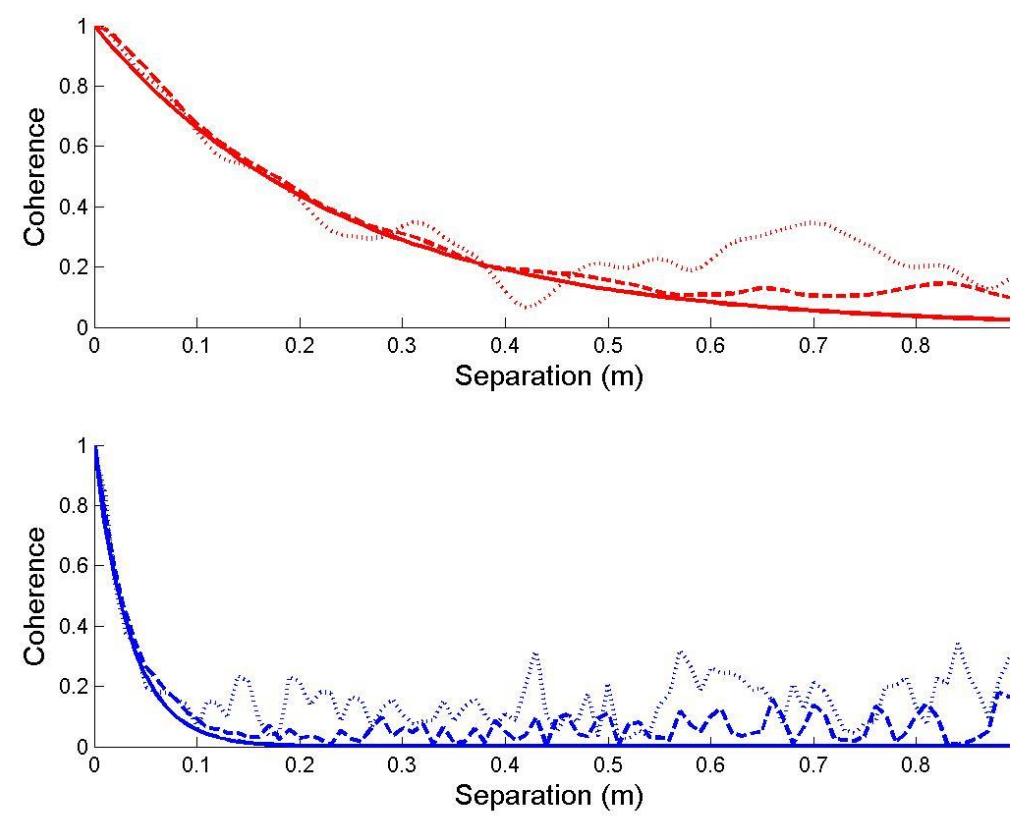

Fig. 6. Spatial coherence of the TBL pressure field as a function of the spatial separation in the streamwise direction (Upper) and in the crosswise direction (Lower). Full line, analytical formula of the Corcos model; dotted line, numerical estimation considering 30 realizations; dash line, numerical estimation considering 900 realizations. 


\section{Results of the sampling method on the nominal test case}

The process described in section 4 is applied to evaluate the ASD function of the panel acceleration at the receiving point. The nominal test case with the Corcos model and 30 realizations are considered. The cut-off wavenumber criterion based on the TBL characteristics (34) is applied. For each realization, the panel acceleration has been obtained using the modal expansion Eq. (29-30). One recalls that the modal forces resulting of the WPF is then calculated analytically. To illustrate the process, one has plotted on Fig. 7 the results of the 30 realizations (grey line). One can observe a relatively large dispersion of the plate response in function of the realizations. The ensemble average of these acceleration responses (i.e. Eq. (22)) is then calculated in order to estimate the ASD function of the panel acceleration. The result (dash line) has been plotted on Fig. 7 .

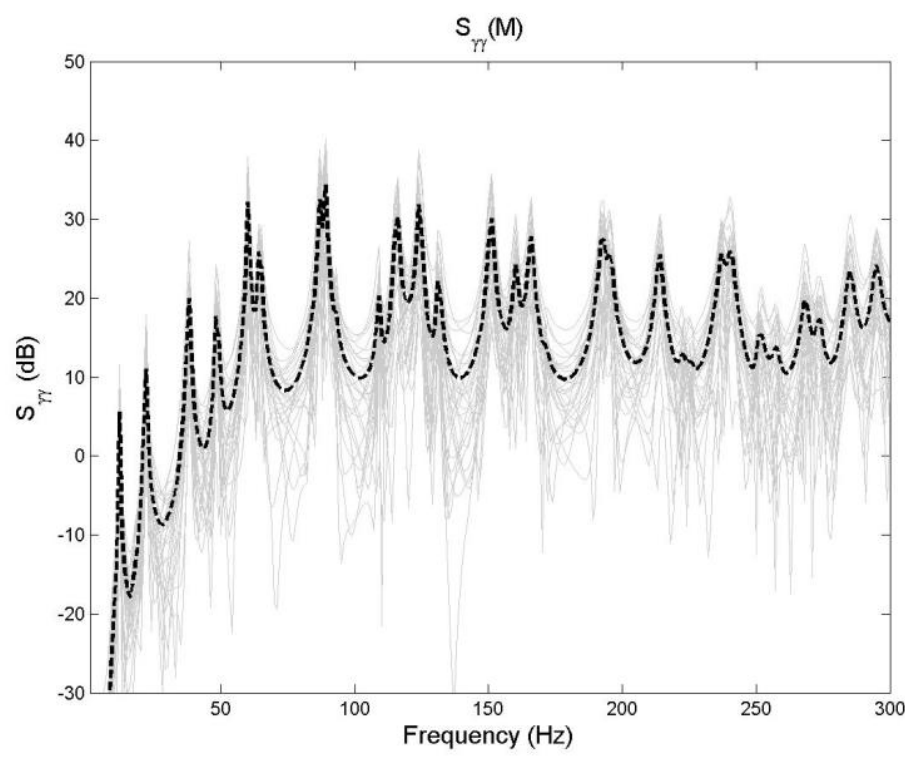

675 Fig. 7. $10 \log _{10}\left[S_{\gamma /}(M)\right]$ (dB, ref. $\left.1 \mathrm{~m} / \mathrm{s}^{2} / \mathrm{Hz}^{0.5}\right)$. Calculations with the sampling method: Grey lines, results of 30 realizations; dashed-dotted line, Results obtained with averaging on the 30 realizations. Calculation parameters: Corcos model, cutoff wavenumber criterion (34) based on the TBL characteristics.

For studying the accuracy of the sampling method, one compares the previous result (dash line) with the result of the sensitivity method (full line) on Fig. 8. A very good agreement between the two calculations can be observed. This indicates that although the panel response of the 30 realizations exhibits an important dispersion, an average over only 30 realizations is sufficient to give a correct estimation of the ASD function of the panel acceleration. 


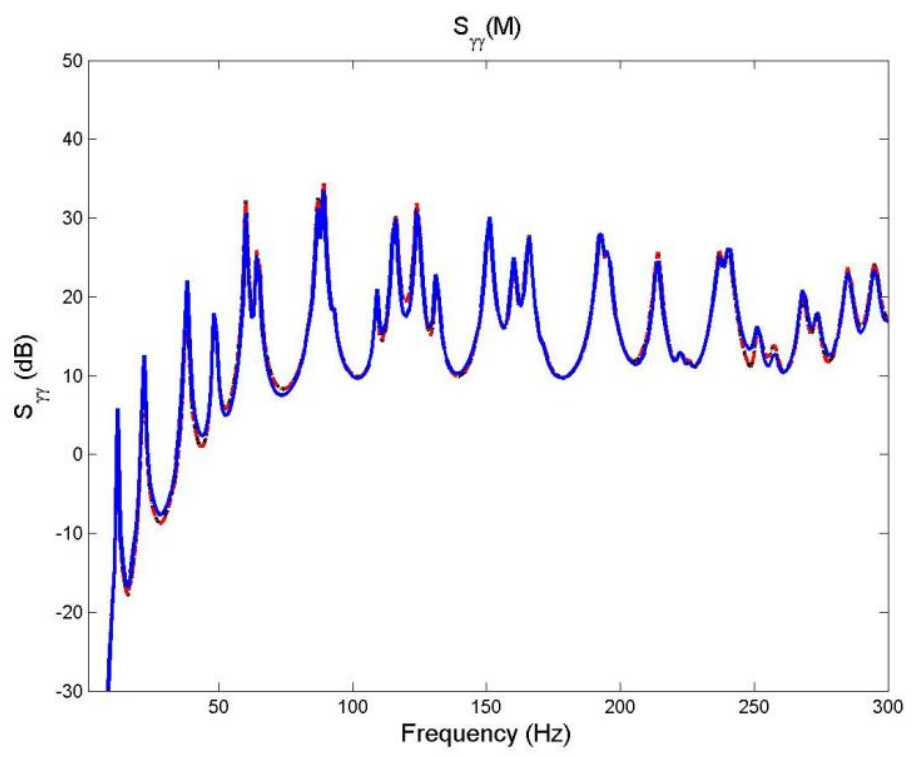

Fig. 8. $10 \log _{10}\left[S_{\gamma /}(M)\right]$ in function of frequency $\left(\mathrm{dB}\right.$, ref. $\left.1 \mathrm{~m} / \mathrm{s}^{2} / \mathrm{Hz}^{0.5}\right)$. Comparison between three calculations: full line, the sensitivity function method (used as reference); dash line: the sampling method with the modal forces calculated analytically; dashed-dotted line, the sampling method with the modal forces estimated using the fine mesh (25 x 9). Calculation parameters: Corcos model, cutoff wavenumber criterion (34) based on the TBL characteristics.

For this first result of the sampling method, Eq. (17) were used to synthetize the WPF and the modal forces were calculated analytically with Eq. (30). This is appropriate for academic cases. For more complex cases, the WPF will be defined on a point mesh and it will be introduced in the numerical model of the panel. In the literature, different authors considering different spatial methods ([24], [25], [28]) already showed that the mesh of a finite element model should be defined carefully in this case for describing both, the structure behavior and the aerodynamic field. For studying the influence of the definition of the WPF on a discretized mesh in the framework of the proposed approach, let us considered a first mesh of points on the plate. This will be called the fine mesh and it is defined by the spatial resolutions $\Delta x$ and $\Delta y$ defined by (as proposed in Ref. [25]):

$$
\Delta x=\frac{\pi}{\overline{k_{x}^{\gamma 2}}} \text { and } \Delta y=\frac{\pi}{\frac{k_{y}^{\gamma}}{\gamma}}
$$

where $k_{x}^{\gamma 2}$ and $k_{y}^{\gamma}$ are defined by the criteria (34) and (35), respectively. 
For the nominal test case, the mesh is composed of 25 points in the streamwise direction and 9 points in the crosswise direction. The modal forces used to estimate the plate acceleration (29) can then be approximated using Eq. (31). The result of the sampling method considering the modal forces estimated on the fine mesh has been plotted (dashed-dotted line) on Fig. 8. One can observe that the discretization of the WPF using this fine mesh does not introduce significant discrepancy which is consistent with the works proposed in the literature.

\section{Influence of the cut-off wavenumber criterion and the mesh size}

Now, the accuracy of the sampling method is studied in function of the cut-off wavenumber criterion and the mesh size. To do that, a second mesh called the coarse mesh is considered with the spatial resolution in the streamwise direction defined by:

$$
\Delta x=\frac{\pi}{k_{x}^{\gamma 1}}, .
$$

where $k_{x}^{\gamma 1}$ is defined by the criterion (33).

For the nominal test case, this coarse mesh is composed of 14 points in the streamwise direction. The spatial resolution in the crosswise direction remains unchanged compared to the fine mesh.

Results of the sampling method are compared to the sensitivity method on Fig. 9 when considering the coarse mesh and the two cutoff wavenumber criteria (33) and (34). Fig. 9a corresponds to the Corcos model whereas as Fig. $9 \mathrm{~b}$ corresponds to the Chase model.

When the cut-off wavenumber criterion (34) based on the convective wavenumber is considered, one notices that the discrepancies are generally less than $2 \mathrm{~dB}$ when the fine mesh is considered, whereas large discrepancies above around $180 \mathrm{~Hz}$ can be observed when the coarse mesh is used. This is observed for the two WPF models. Although the contributions of the convective ridge are filtered by the panel and may be neglected as shown in section V.C, a fine description of them is required in order to obtain good convergence of the calculation. In contrary, when considering the coarse mesh and the cut-off wavenumber criterion (33) based on the flexural wavenumber, a good accuracy is observed on the whole frequency of interest, for both WPF models. This result confirm that the effect of the convective ridge is negligible on the panel vibration for the present case. Moreover, the coarse mesh is sufficient for 
735 estimating the panel response because the pressure field of each realization (as shown on Fig. 5 b) varies slowly when the criterion (33) is considered (contrary to the rapid variations of the pressure field when the criterion (34) is considered, as shown in Fig. 5a). This explains why the results converge with a coarse mesh when the criterion (33) is considered rather than the criterion (34).

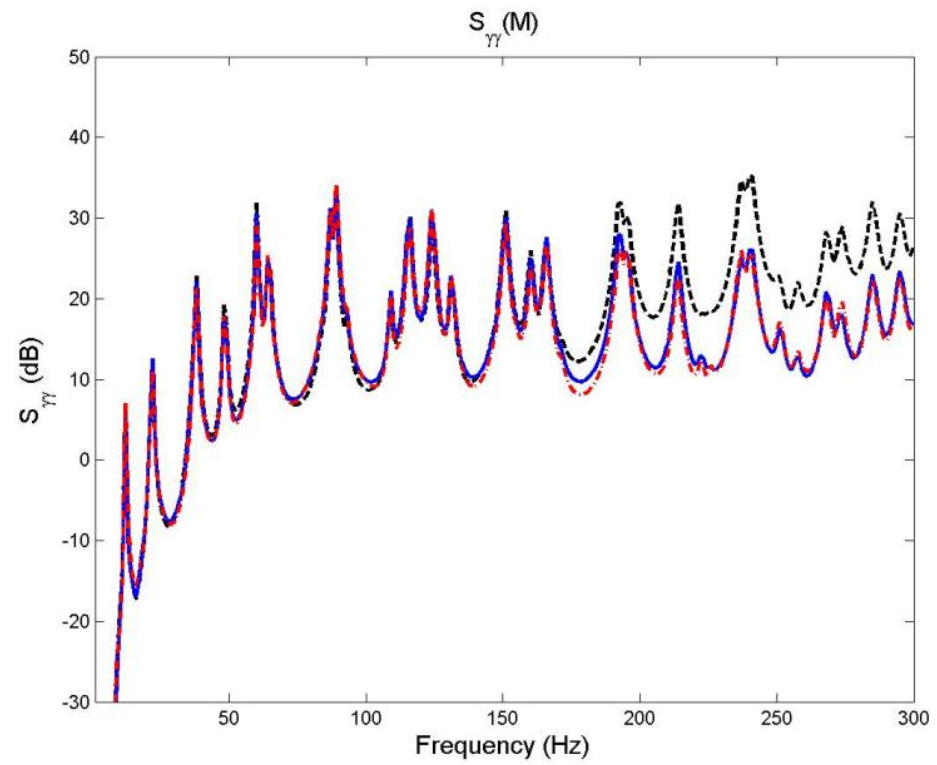

(a)

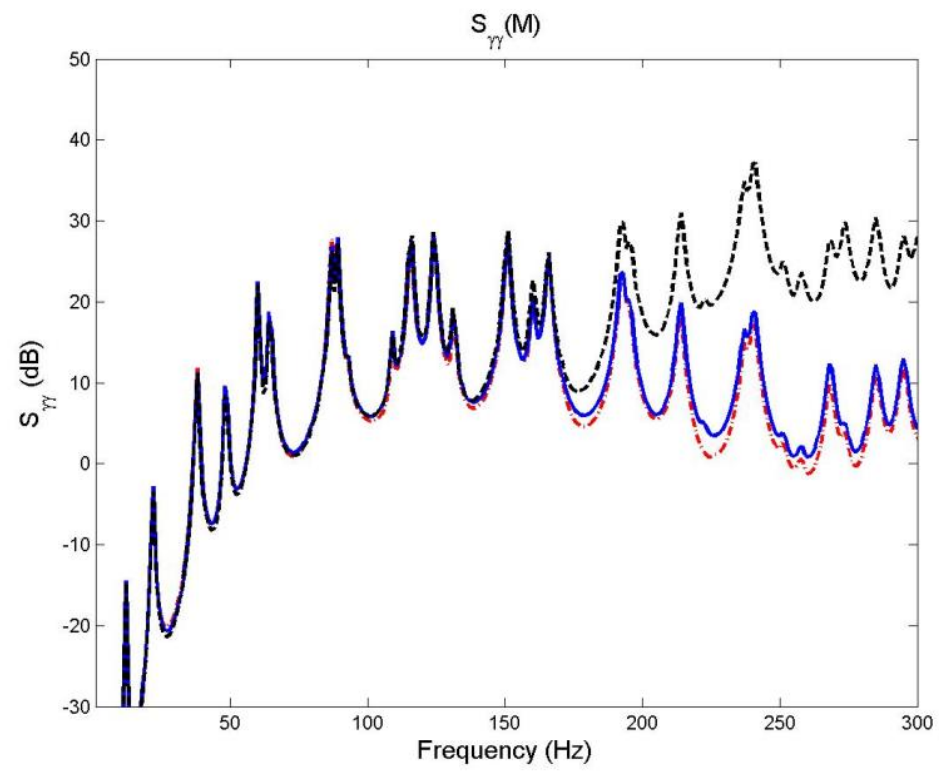

(b)

$740 \quad$ Fig $9.10 \log _{10}\left[S_{\gamma \gamma}(M)\right]$ in function of frequency $\left(\mathrm{dB}\right.$, ref. $\left.1 \mathrm{~m} / \mathrm{s}^{2} / \mathrm{Hz}^{0.5}\right)$. Results with two different models of WPF: (a), Corcos; (b), Chase. Full line, the sensitivity function method (used as reference); dash line, the sampling method considering the coarse mesh (14 x 9) and the cutoff wavenumber criterion (34) based on the TBL characteristics; dashed-dotted line: the sampling method considering the coarse mesh $(14 \times 9)$ and the cutoff wavenumber criterion (33) based on the plate characteristics. 
Thus, when the panel filtering effect is dominant, there are two advantages in reducing the wavenumber space:

- first, it reduces the number of wall plane waves to be considered (i.e. card( $\Pi)$ ) in the process of synthetizing the WPF (17). As the computing time of this process is directly proportional to the number of wall plane waves considered, the time saving can be easily deduced when using (33) instead of (34). However, it should be pointed out that this process, which consists in performing a summation, consumes relatively little time;

- second, as it was observed previously, it permits to consider a coarser mesh than if the wavenumber space were not reduced. In the case of a panel represented by a Finite Element model, the size of the elements can be defined with a criterion on the panel characteristics and not the TBL ones. The number of degrees of freedom, and by consequence, the FEM computing times, can be significantly reduced.

We should however underline that this panel filtering effect is not always dominant and it should be considered carefully. It is for instance the case when the frequency range of interest is close to the hydrodynamic coincidence frequency. The sampling method can however always be applied using the criterion (34) and a fine mesh described with (37).

\section{Influence of the number of realizations}

765

To evaluate the influence of the number of realizations on the accuracy of the sampling method, the discrepancies with the sensitivity function method are plotted in Fig. 10 for different numbers of realizations. An optimal compromise should be found between reasonable computing times and acceptable discrepancies. For the presented case, with 30 realizations, the average over the frequency range of the absolute discrepancies is less than $1 \mathrm{~dB}$ between the sensitivity function method and the sampling method. This seems acceptable from the practical point of view. 


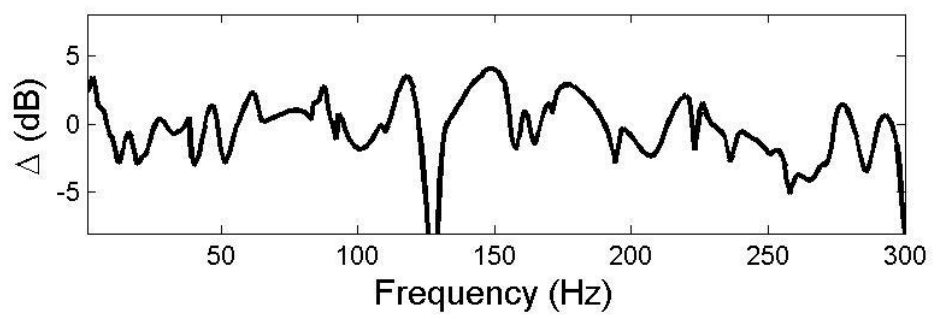

(a)

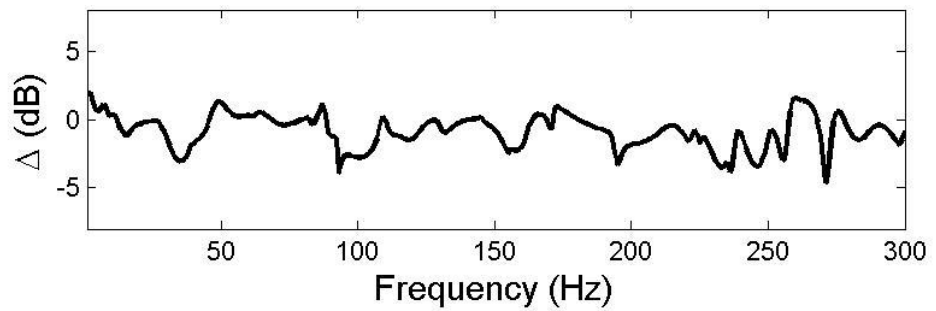

(b)

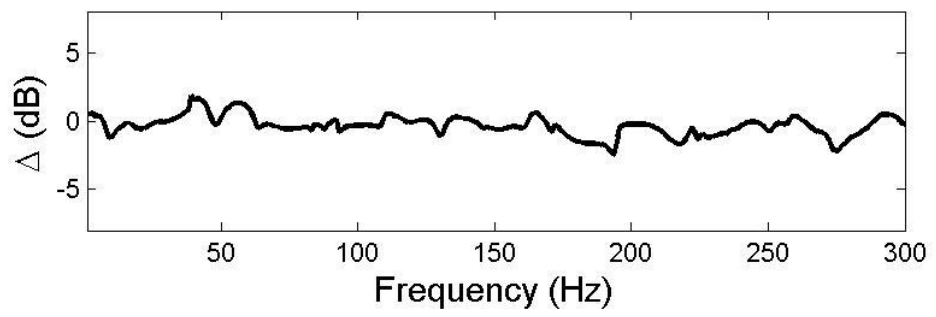

(c)

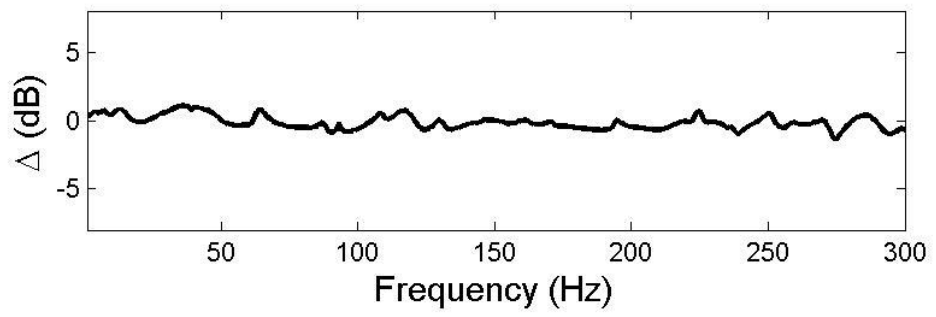

(d)

Fig. 10. Difference of $10 \log _{10}\left[S_{\gamma /}(M)\right]$ between the sensitivity function method and the sampling method as a function of frequency for different number of realization: (a), 3 realizations; (b), 10 realizations; (c), 30 realizations; (d), 100 realizations.

\section{Computation times}

780

The calculations presented in this paper have been performed using the MATLAB software on a standard personal computer (Intel Core i5 $3.2 \mathrm{GHz}, 8 \mathrm{Go}$ Ram). When the cut-off wavenumber criterion (34) were applied, 17738 points in the wavenumber space (or wall plane waves) were considered. For the sensitivity method, 815 seconds were used to calculate the entire spectrum whereas 331 seconds were used by the sampling method with the fine mesh. When the cut-off wavenumber criterion (33) were applied, 9604 points in the wavenumber space were 
considered. The computation time are reduced to 689 seconds for the sensitivity method and 114 seconds for the sampling method with the coarse mesh. This computing time have been given for indication but they should be relativized. It can be strongly depend on the vibroacoustic model used to describe the panel. When a commercial software is used, an important parameter can be the number of load cases being considered. In the case of the sensitivity method, this one corresponds to the number of wall plane waves whereas for the sampling method, it is the number of realizations. It is an important advantage of the sampling method compared to the sensitivity approach. Moreover, more than $90 \%$ of the computation time of the sampling method corresponds to the synthetize of the WPF with (17). The summation which appears in this equation is performed with a FOR loop in the MATLAB program, which is time consuming. The use of a programming language such as FORTRAN or C could certainly save computing time.

Even if the numerical process related to the sampling process is not fully optimized, the computing times given previously clearly show that the sampling method permits to save computing times compared to the sensitivity method.

\section{Influence of the convective velocity and the panel thickness}

To verify the accuracy of the sampling approach in function of different physical parameters, we are going to modify two parameters of the nominal case: the convective velocity and the panel thickness. The others parameters will remain unchanged. The Chase model and 30 realizations are considered in this section. The modification of these parameters will change the hydrodynamic coincidence frequency and the interaction between the pressure fluctuation of the TBL and the panel response.

\section{- Influence of the convective velocity}

Calculations with the sensitivity method and the sampling method have been performed for two convective velocities: $93 \mathrm{~m} / \mathrm{s}$ and $25 \mathrm{~m} / \mathrm{s}$. For the first one, the hydrodynamic coincidence 815 frequency is $300.1 \mathrm{~Hz}$. At $300 \mathrm{~Hz}$, the higher frequency range of interest, the flexural wavenumber is close to the convective wavenumber. The cut-off wavenumber in the streamwise direction can then be defined independently with criterion (33) or (34) which ensure to describe correctly the panel behavior and the WPF. A good agreement has been obtained between the sensitivity approach and the sampling approach (results no shows here). For the second convective velocities (i.e. $25 \mathrm{~m} / \mathrm{s}$ ), the hydrodynamic coincidence frequency is $21.7 \mathrm{~Hz}$. 
The cut-off wavenumber in the streamwise direction given by the criterion (34) is equal to 90.5 $\mathrm{rad} / \mathrm{m}$ and the fine mesh in the streamwise direction is composed of 45 points. The results are plotted on Fig. 11. We can notice a good agreement between the different results. Some slight discrepancies can be observed between the sensitivity approach (used as reference) and the sampling approach when considering the criterion (33). This can be attributed to the fact that the panel filtering effect is not sufficient to vanish completely the effect of the convective ridge. The results remains however acceptable from a practical point of view.

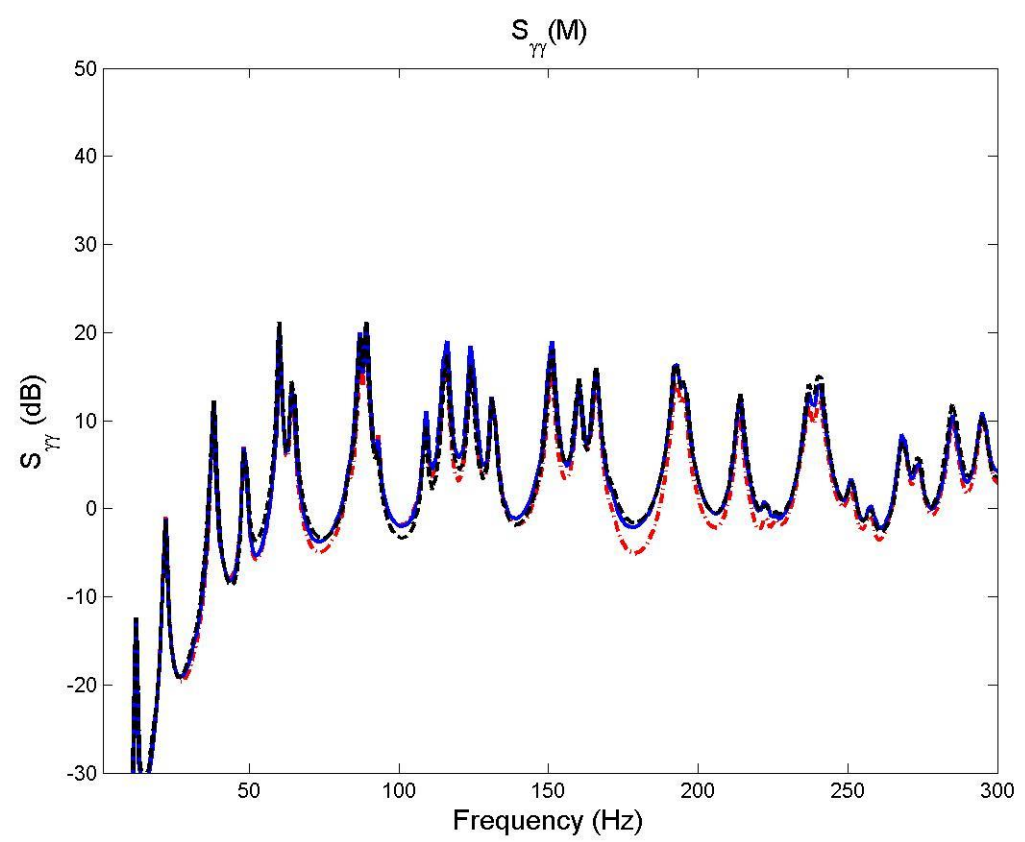

Fig 11. $10 \log _{10}\left[S_{\gamma \gamma}(M)\right]$ in function of frequency $\left(\mathrm{dB}\right.$, ref. $\left.1 \mathrm{~m} / \mathrm{s}^{2} / \mathrm{Hz}^{0.5}\right)$. Results for the convective velocity, $U_{c}=25 \mathrm{~m} / \mathrm{s}$. Chase Model. Full line, the sensitivity function method (reference); dash line, the sampling method considering the fine mesh (45 x 9) and the cutoff wavenumber criterion (34) based on the TBL characteristics; dashed-dotted line: the sampling method considering the coarse mesh (14 x 9) and the cutoff wavenumber criterion (33) based on the plate characteristics.

\section{- Influence of the panel thickness}

Now, the convective velocity is fixed to $25 \mathrm{~m} / \mathrm{s}$ and two panel thickness are considered: 6 $\mathrm{mm}$ and $1.5 \mathrm{~mm}$. The values of the hydrodynamic coincidence frequency, the cut-off wavenumber defined by the criterion (33) and the number of points of the coarse mesh in the streamwise direction are respectively, $10.8 \mathrm{~Hz}, 17.2 \mathrm{rad} / \mathrm{m}, 10$ for the $6 \mathrm{~mm}$ thick panel, and $43.3 \mathrm{~Hz}, 34.4 \mathrm{rad} / \mathrm{m}, 18$ for the $1.5 \mathrm{~mm}$ thick panel. The results shown on Fig. 12 indicates 
again a good agreement between the different calculations. When using the coarse mesh, the differences are slightly more important for the $6 \mathrm{~mm}$ thick panel than for the $1.5 \mathrm{~mm}$ thick panel. It is always an effect of the weakness of the panel filtering effect. It should however notice that the size of the coarse mesh (i.e. 90 points) is significantly lower than the one of the fine mesh (i.e. 405 points). For this case, it can be accepted to lose slightly in accuracy in order to decrease the number of degree of freedom of the vibro-acoustic model.

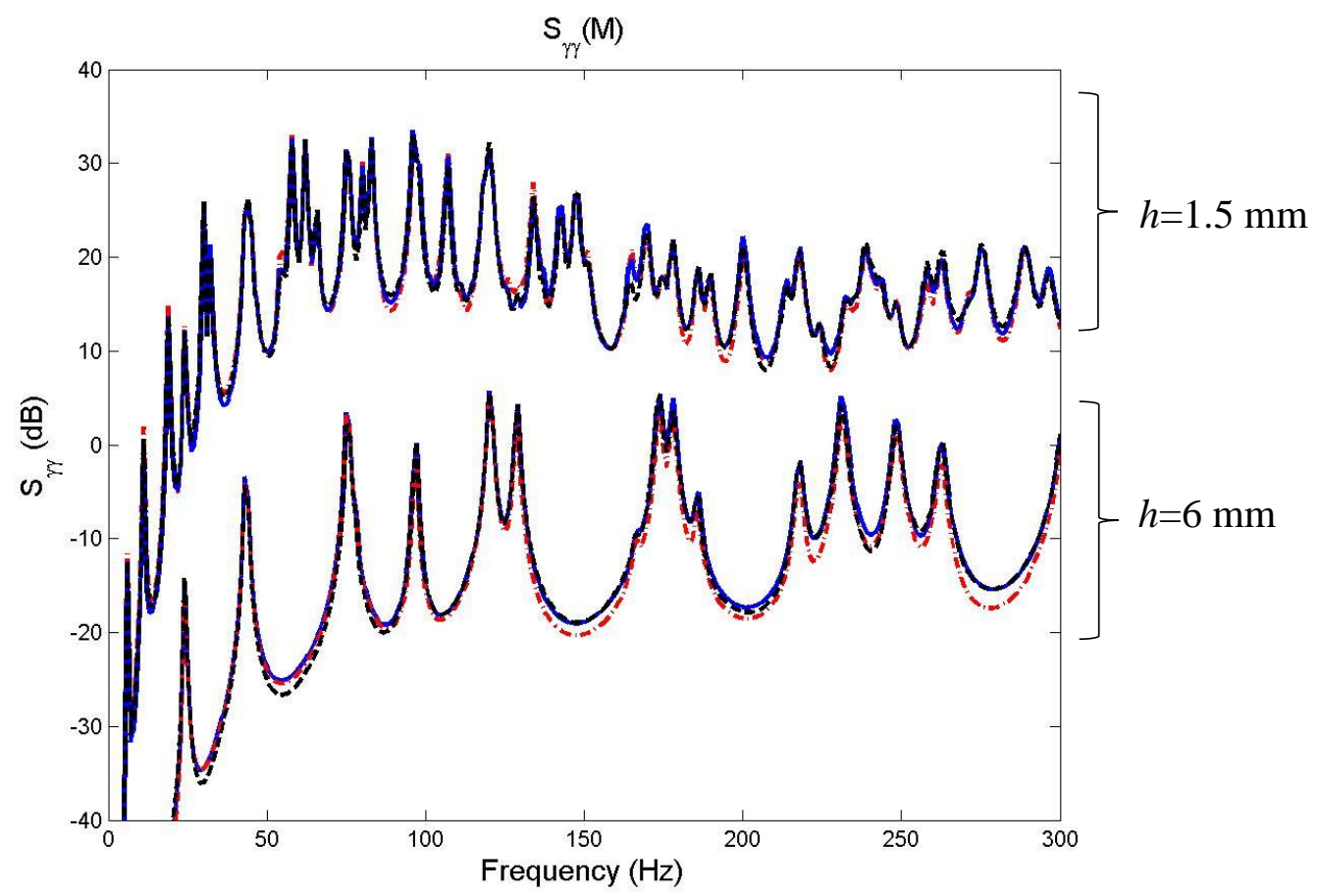

Fig 12. Same results than Fig. 11 with two different plate thickness: upper curves, $h=1.5 \mathrm{~mm}$; lower curves, $h=6 \mathrm{~mm}$.

\section{EXAMPLE OF APPLICATION ON A STIFFENED PANEL}

Now let us illustrate one interest of the sampling approach with an application on a complex panel. Indeed, one has shown that the number of load cases which should be considered in the sensitivity method corresponds to the number of wall plane waves whereas for the sampling method, it corresponds to the number of realization which is small compared to the number of wall plane waves. The number of forced responses which should be calculated with the vibroacoustic model is then relatively small when using the sampling method. This can be of high interest when the vibro-acoustic calculations are performed by a commercial software for which 
the calculation process cannot be easily modified. For highlighting this interest, one considers a complex panel composed by a rectangular plate orthogonally stiffened by ribs regularly spaced. The rectangular plate corresponds to the one of the nominal case (see Tab. 1). We recall that the plate is assumed to be simply-supported at its four edges and it is excited by a turbulent air flow with the flow direction parallel to the longest edges of the plate. The rib cross-section is rectangular, $3 \mathrm{~mm}$ thick and $60 \mathrm{~mm}$ high. The rib spacing in the direction of the longest plate edges is $500 \mathrm{~mm}$ whereas it is of $300 \mathrm{~mm}$ in the other direction. The plate and the ribs are both made of aluminum (see Tab. 1). The characteristics of the flow considered for this application are those of the nominal case (see Tab. 1) and the Chase model is used to describe the WPF.

The stiffened panel is modelled using the finite element method and the MSC/NASTRAN software. The plate and the ribs are modelled with 2D shell elements (i.e. CQUAD4 elements with PSHELL properties) as shown on Fig. 13. A criterion of six elements by flexural wavelength at $300 \mathrm{~Hz}$ were considered. A direct analysis (i.e. SOL 108) in the MSC/NASTRAN software allows us estimating the forced response of the stiffened panel excited by a deterministic load. The sampling method is then used to estimate the panel response to the WPF induced by the TBL. The receiving point of interest is $M^{\prime}$ of coordinates $\left(x_{M^{\prime}}, y_{M^{\prime}}\right)=(0.14 \mathrm{~m}, 0.22 \mathrm{~m})$ which corresponds to the node of the mesh closest the point $M$ of the nominal case of Sec. V.

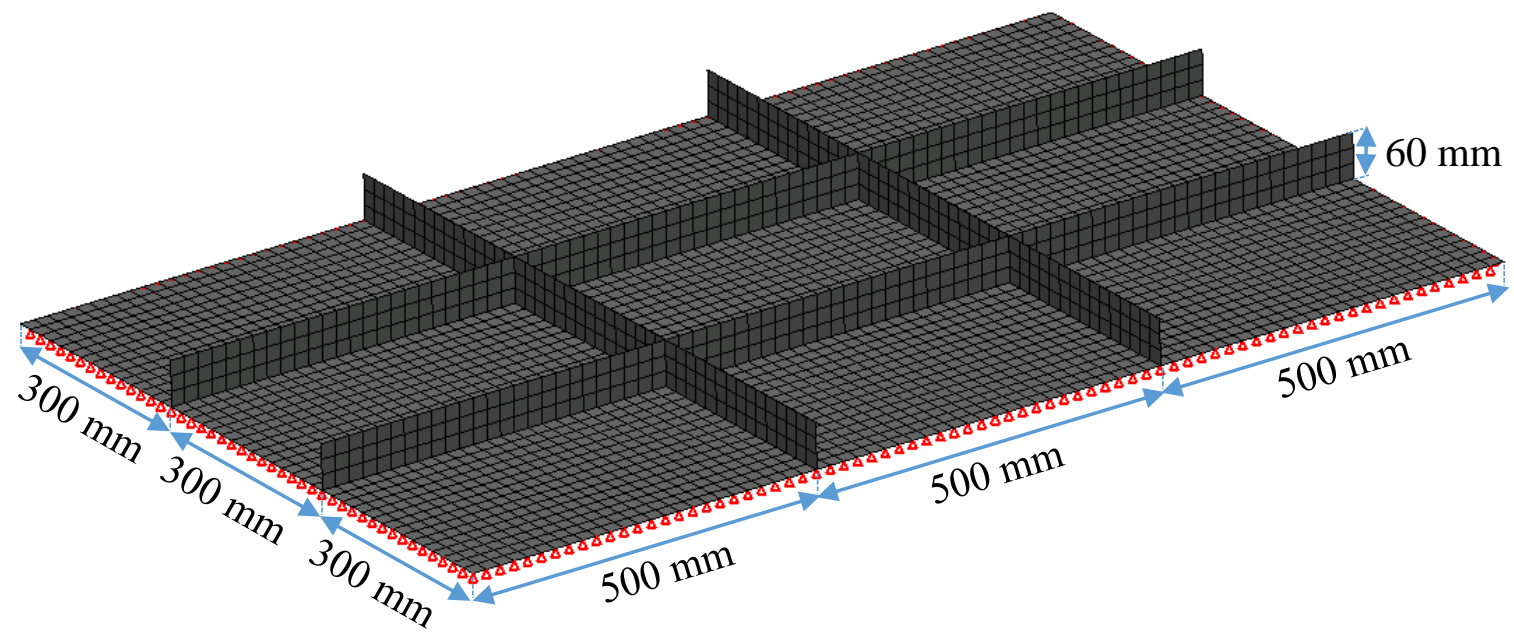

Fig. 13. Finite element mesh of the stiffened plate (view opposite side to the flow). 4216 nodes, 4095 quadrilateral elements. 
The numerical process were decomposed in 3 steps:

- the WPF corresponding to 30 realizations were synthetized using Eq. (21) and a MATLAB program. The cut-off wavenumber criterion (33) was considered for this application as the ribbed panel was stiffer than the panel of the nominal case. For each realization, the WPF was exported in an ASCII file using the MSC/NASTRAN input data file format. The size of each file was $128 \mathrm{Mo}$;

- A direct analysis was performed with the MSC/NASTRAN solver to calculate the forced responses induced by the WPF of the different realizations. To do this, multi-load cases were managed in the software by defining different SUBCASE. The 30 files containing the WPF of the 30 realizations were read in 170 seconds. The calculations were then performed in 90 seconds on the computer described previously. The forced responses at the receiving point $M$ ' were exported in a PCH ASCII file;

- The PCH file of 8 Mo were read with a MATLAB program and the ensemble average over the 30 realizations (i.e. Eq. (22)) were performed to evaluate the ASD function of the panel acceleration at point $M$ ' induced by the TBL excitation.

In order to validate this numerical process, the calculations were performed first considering the unstiffened panel (which corresponds to the nominal case of Sec. V). The results are compared on Fig. 14 with the results of the sensitivity method. A good agreement between the two calculations can be globally observed on the whole frequency range that validate the numerical process. Above $250 \mathrm{~Hz}$, slight shifts of the peaks can be noticed that can be attributed to the finite element discretization of the vibro-acoustic problem. 


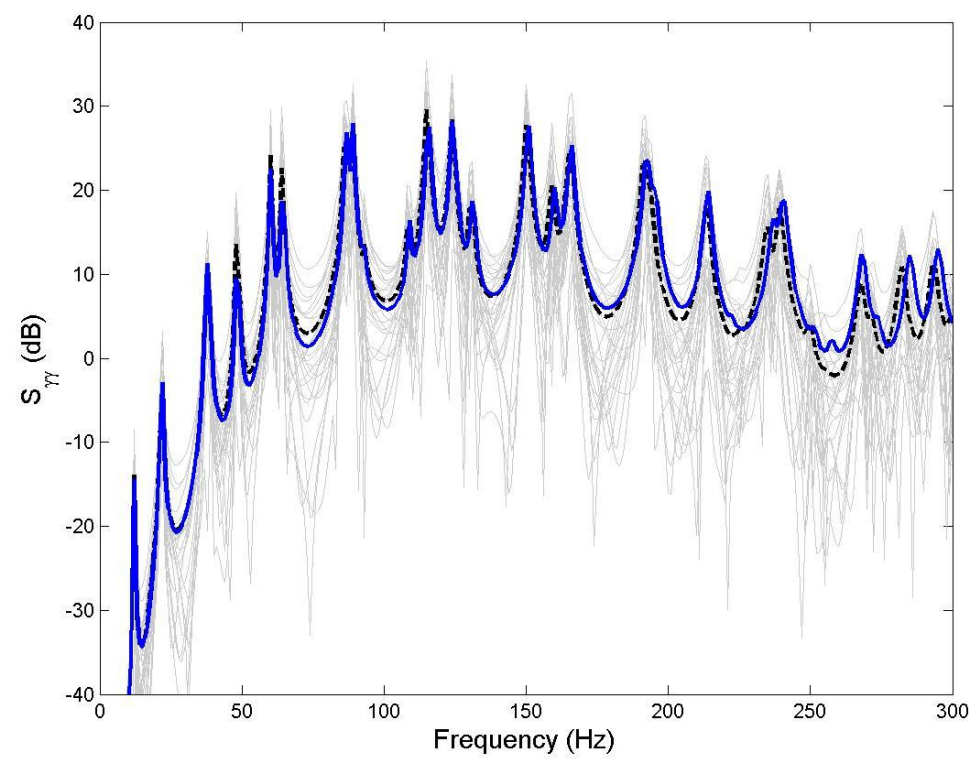

Fig. 14. $10 \log _{10}\left[S_{\gamma \gamma}\right]$ in function of frequency $\left(\mathrm{dB}\right.$, ref. $\left.1 \mathrm{~m} / \mathrm{s}^{2} / \mathrm{Hz}^{0.5}\right)$ for the panel without stiffeners. Full line, the sensitivity method at point $M$; dashed-dotted line, the sampling method at point $M^{\prime}$ ' using MSC/NASTRAN FEM code (with averaging on 30 realizations). Grey lines, results of each one of the 30 realizations. Calculation parameters: Chase model, $U_{c}=50 \mathrm{~m} / \mathrm{s}$, cutoff wavenumber criterion (33) based on the plate characteristics.

The calculations were then achieved for the stiffened plate. We can underline that it was not necessary to perform again the first step of the numerical process described above because the characteristics of the flow are the same for the two cases. The WPF are then unchanged. The results of the sampling method are plotted on Fig. 15. Compared to the unstiffened plate, we observe that the vibratory levels are globally lower and the first peak appears at a higher frequency. The fact that the static stiffness of the ribbed plate is higher than the one of the bare plate explains this behavior. Moreover, some groups of peaks appears on the spectrum of the stiffened panel. These groups can be related to the behavior of an orthotropic plate [46]. Indeed, as the plate is stiffened by two ribs in one direction and three ribs in the other direction, the flexural stiffness is lower in one direction than the other one. The panel has then a behavior equivalent to an orthotropic plate in the low frequency range [46]. In higher frequencies, more complex phenomena like the propagation of Bloch-Floquet waves [40] would influence the panel behavior. It is however outside the scoop of the present application which has been proposed for highlighting the interest of the sampling approach. 


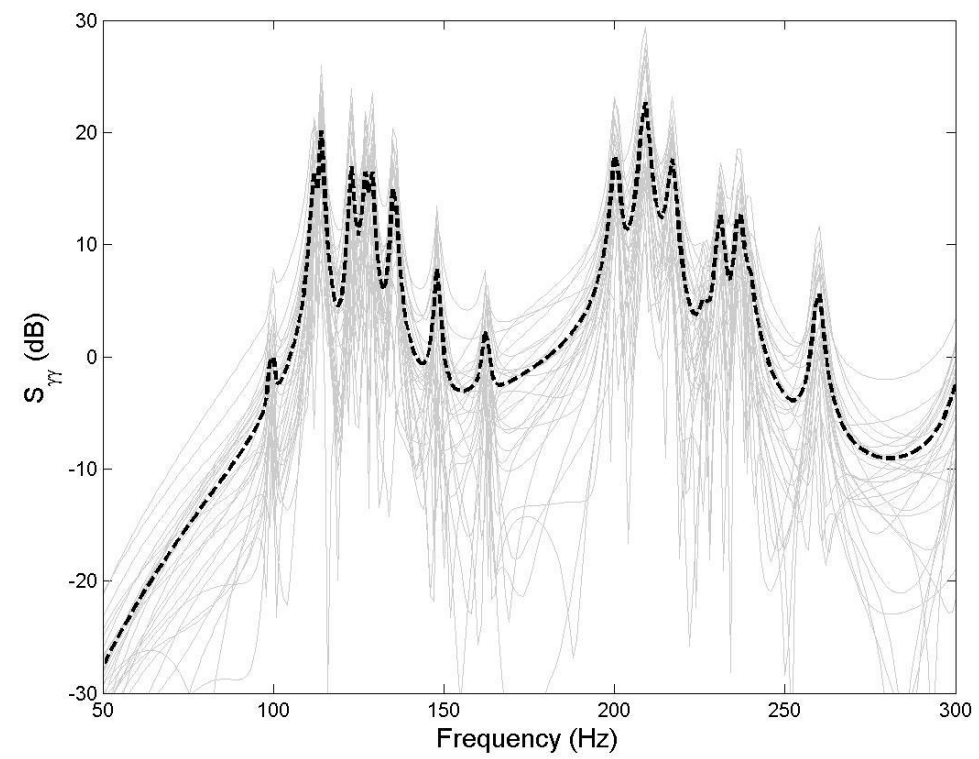

Fig. 15. $10 \log _{10}\left[S_{\gamma /}\left(M^{\prime}\right)\right]$ in function of frequency $\left(\mathrm{dB}\right.$, ref. $\left.1 \mathrm{~m} / \mathrm{s}^{2} / \mathrm{Hz}^{0.5}\right)$ for the stiffened panel. Dashed-dotted line, the sampling method using MSC/NASTRAN FEM code (with averaging on 30 realizations). Grey lines, results of each one of the 30 realizations.

Calculation parameters: Chase model, $U_{c}=50 \mathrm{~m} / \mathrm{s}$, cutoff wavenumber criterion (33) based on the plate characteristics.

\section{CONCLUDING REMARKS}

The numerical process proposed in this paper is based on two main characteristics:

- (a) on the formulation of the random excitation problem expressed in the frequencywavenumber domain. This permits using the analytical expression of the wall pressure spectrum directly in the wavenumber of the well-known models in the literature (Corcos [2], Chase [3], Smol'yakov [4], etc.). Moreover, the truncation of the wavenumber space can be achieved easily with cut-off wavenumbers defined from the panel characteristics when the panel filtering effect is predominant. This permits taking advantage of this well-known effect to optimize the numerical process. Indeed, when the cut-off wavenumber is based on the panel characteristics, it is not necessary to consider a fine spatial description of the pressure field, as discussed in Sec. V. We should however emphasize that the filtering effect of the structure is not always enough 
955 important to vanish the contributions of the convective ridge. This depends in particular on the frequencies of interest, the panel boundary conditions, and the WPF model. In this case, the criterion (34) based on the TBL characteristics and a fine spatial description of the pressure field (see Eq. ((37)) should be considered;

- (b) on the interpretation of the WPF as uncorrelated wall plane waves. This provides 960 a simple way of synthetizing the realizations of the WPF corresponding to the CSD function. Indeed, Eq. (17) can be applied directly by considering the ASD functions of the wave amplitudes defined by Eq. (14). The WPF is then simply obtained by a summation of the wall plane waves defined from the CSD function of the wall pressure. The process is then easily implemented in a computer program and is not very time consuming.

The numerical application showed than the sampling method gives a good estimation of the panel response even when a relatively small number of realizations (typically 30) are considered. This means that only a few deterministic load cases should be considered in the vibroacoustic calculations. This permits to save computing times compared to the classical approaches like the sensitivity method. As it has been highlighted on a stiffened panel, the process can be used with any commercial vibroacoustic software applications based on an element-based method. In this situation, a pre-processing program can be used to synthetize the pressure field on each node of the mesh for each load case (corresponding to one realization) and to export them in an input datafile adapted for the vibroacoustic software. As the number

975 of load cases is relatively small, the size of this input datafile should also remain relatively small. After running the vibroacoustic code, a post-processing program read the output datafile containing the system responses and performed the ensemble averaging for the different load cases (i.e. realizations). The process proposed is therefore non-intrusive in the vibroacoustic software.

The proposed numerical process has been applied to estimate the vibratory response of a vibrating panel. From a theoretical point of view, there is no obstacle for extending it to predict the pressure radiated by the panel excited by a TBL. Indeed, the formulation in the frequencywavenumber domain of the radiated pressure can be achieved similarly to the panel vibration 985 [39]. It is then easily to show that the ASD function of the radiated pressure can be estimated from the superposition of the uncorrelated wall pressure plane waves as for the panel vibration (see Sec. III). From a practical point of view, it remains however to study the convergence of 
the process in function of the number of realization and to define cut-off wavenumber criteria adapted to the evaluation of the radiated noise.

990

\section{ACKNOWLEDGMENTS}

This work was carried out in the framework of the LabEx CeLyA ("Centre Lyonnais d'Acoustique", ANR-10-LABX-60).

\section{REFERENCES}

[1] Flinovia - Flow induced noise and vibrations issues and aspects: A focus on measurement, modeling, simulation and reproduction of the flow excitation and flow induced response (Springer International Publishing Switzerland, 2015, pp. 357)

[2] G. M. Corcos, "Resolution of pressure in Turbulence," J. Acoust. Soc. Am. 35, $192-$ 199 (1963).

[3] D.M. Chase, "The character of the turbulent wall pressure spectrum at subconvective wavenumbers and a suggested comprehensive model," J. Sound Vib. 112, 125-147 (1987).

[4] A.V. Smol'yakov and V.M. Tkachenko, "Model of a field of pseudosonic turbulent wall pressures and experimental data," Sov. Phys. Acoust. 37, 627-631 (1991)

[5] Y. Hwang, W. Bonness, and S. Hambric, "Comparison of semi-empirical models for turbulent boundary layer wall pressure spectra," J. Sound Vib. 319, 199-217 (2009).

[6] M. Goody, "Empirical spectral model of surface pressure fluctuations," AIAA J. 42, 1788-1794 (2004).

[7] Y. Rozenberg, G. Robert, et S. Moreau, "Wall-Pressure Spectral Model Including the Adverse Pressure Gradient Effects," AIAA J. 50, 2168-2179 (2012).

[8] E. Ciappi, F. Magionesi, S. De Rosa, F. Franco, "Hydrodynamic and hydroelastic analyses of a plate excited by the turbulent boundary layer," J. Fluids Struc. 25, 321342 (2009). 
[9] W. Bonness, D. Capone, and S. Hambric, "Low-wavenumber turbulent boundary layer wall-pressure measurements from vibration data on a cylinder in pipe flow," J. Sound Vib. 329, 4166-4180 (2010).

[10] W. R. Graham, “A comparison of models for the wavenumber-frequency spectrum of turbulent boundary layer pressures," J. Sound Vib. 206, 541-565 (1997).

[11] W. A. Strawderman, "Turbulence-induced plate vibrations: An evaluation of finiteand infinite-plate models," J. Acoust. Soc. Am. 46, 1294-1295 (1969).

[12] W. A. Strawderman and R. A. Christman, "Turbulence-induced plate vibrations: Some effects of fluid loading on finite and infinite plates," J. Acoust. Soc. Am. 52, 15371552 (1971).

[13] H. G. Davis, "Sound from turbulent boundary layer excited panel," J. Acoust. Soc. Am. 49, 878-889 (1971).

[14] W. R. Graham, "Boundary layer induced noise in aircraft. Part I: The flat panel model," J. Sound Vib. 192, 101-120 (1995).

[15] S. H. Ko and H. H. Schloemer, "Flow noise reduction techniques for a planar array of hydrophones," J. Acoust. Soc. Am. 92, 3409-3424 (1992).

[16] S. H. Ko and H. H. Schloemer, "Calculations of turbulent boundary layer pressure fluctuations transmitted into a viscoelastic layer,” J. Acoust. Soc. Am. 85, 1469-1477 (1989).

[17] D. Mazzoni, “An efficient approximation for the vibro-acoustic response of a turbulent boundary layer excited panel," J. Sound Vib. 264 , 951-971 (2003).

[18] M. L. Rumerman, "Estimation of broadband acoustic power due to rib forces on a reinforced panel under turbulent boundary layer-like pressure excitation. I. Derivations using strong model,” J. Acoust. Soc. Am. 109, 563-575 (2001).

[19] M. L. Rumerman, "Estimation of broadband acoustic power due to rib forces on a reinforced panel under turbulent boundary layer-like pressure excitation. II. Applicability and validation,” J. Acoust. Soc. Am. 109, 576-582 (2001).

[20] M.L. Rumerman, "Estimation of broadband acoustic power radiated from a turbulent boundary layer-driven reinforced finite plate section due to rib and boundary forces," J. Acoust. Soc. Am. 111, 1274-1284 (2001).

[21] E. Ciappi, S. De Rosa, F. Franco, P. Vitiello, M. Miozzi, "On the dynamic behavior of composite panels under turbulent boundary layer excitations," J. Sound Vib. 364, 77109 (2016). 
[22] I. Harari, M. Slavutin, and E. Turkel, "Analytical and Numerical Studies of a Finite Element PML for the Helmholtz Equation,” J. Comp. Acoust. 8, 121-137 (2000).

[23] D. W. Herrin, T. W. Wu, and A. F. Seybert, "Chapter 8 - Boundary element modelling," Handbook of Noise and Vibration Control (Ed. M. J. Crocker, John Wiley and Sons, New York, NJ, 2007)

[24] “ACTRAN 12.2 User's Guide. Vol. 1: Installation, operations, theory and utilities." (Free Field Technologies S.A, Belgium, 2012).

[25] C. Hong, K.K. Shin, "Modeling of wall pressure fluctuations for finite element structural analysis," 329, 1673-1685 (2010).

[26] F. Franco, S. De Rosa, E. Ciappi, "Numerical approximations on the predictive responses of plates under stochastic and convective loads,” J. Fluids Struc. 42, 296312 (2013).

[27] M.N. Ichchou, B. Hiverniau, B. Troclet, "Equivalent 'rain on the roof' loads for random spatially correlated excitations in the mid-high frequency range," J. Sound Vib. 322, 926-940 (2009).

[28] S. De Rosa, F. Franco, "Exact and numerical responses of a plate under a turbulent boundary layer excitation,” J. Fluids Struc. 24, 212-230 (2008).

[29] E. Ciappi, F. Magionesi, S. De Rosa, F. Franco, "Analysis of the scaling laws for the turbulence driven panel responses”, J. Fluids Struc. 32, 90-103 (2012).

[30] S. De Rosa, F. Franco, E. Ciappi, "A simplified method for the analysis of the stochastic response in discrete coordinates," J. Sound Vib. 339, 359-375 (2015).

[31] J. Lin, W. Zhang, J. Li, "Structural responses to arbitrarily coherent stationary random excitation," Comp. and Struct., 50, 629-633 (1994).

[32] C.C. Caprani, "Application of the pseudo-excitation method to assessment of walking variability on footbridge vibration," Comp. and Struct., 132, 43-54 (2014).

[33] A. Hekmati, D. Ricot, and P. Druault, "Numerical synthesis of aeroacoustic wall pressure fields over a flat plate: generation, transmission and radiation analyses $\mathrm{J}$. Sound Vib. 332, 3163-3176 (2013).

[34] L.E. Wittig and A.K. Sinha, "Simulation of multicorrelated random processes using the FFT algorithm,” J. Acoust. Soc. Am. 58, 630-634 (1975).

[35] W.K. Blake, Mechanics of Flow-Induced Sound and Vibration. Vol. II: Complex FlowStructure Interaction (Academic press, Inc. Orlando, FL, 1986. pp. 497) 
[36] S. Hambric, Y. Hwang, and W. Bonness, "Vibrations of plates with clamped and free edges excited by low-speed turbulent boundary layer flow,” J. Fluid Struct. 19, 93-110 (2004).

[37] T. Miller, J. Gallman, M. Moeller, "Review of turbulent boundary layer models for acoustic analysis," Proceedings of the $49^{\text {th }}$ AIAA Aerospace Sciences meeting, Orlando, Florida, January 2011.

[38] W. A. Strawderman, "Wavevector-Frequency Analysis with Applications to Acoustics,” Technical Report No. 8209, NUSC (1988), pp. 244.

[39] C. Maury, P. Gardonio, and S. J. Elliott, "A wavenumber approach to modelling the response of a randomly excited panel, Part I : General Theory,” J. Sound Vib. 252, 83 -113 (2002).

[40] L. Maxit and V. Denis, "Prediction of flow induced sound and vibration of periodically stiffened plates," J. Acoust. Soc. Am. 133, 146-160 (2013).

[41] F. Birgersson, N.S. Ferguson and S. Finnveden, "Application of the spectral finite element method to turbulent boundary layer induced vibration of plates," J. Sound Vib. 259, 873-891 (2003).

[42] A.D. Pierce, Acoustics: An Introduction to Its Physical Principles and Applications (Acoustical Society of America, Woodbury, NY, 1989, pp.678).

[43] D.M. Chase, "Modeling the Wavevector-Frequency Spectrum of Turbulent Boundary Layer Wall Pressure,” J. Sound Vib. 70, 29-67 (1980)

[44] N.C. Martin, P. Leehey, "Low wavenumber wall pressure measurements using a rectangular membrane as a spatial filter,” J. Sound Vib. 52, 95-120 (1977)

[45] A. Caiazzo, R. D’Amico, W. Desmet, “A generalized corcos model for modelling turbulent boundary layer wall pressure fluctuations," J. Sound Vib. 372, 192-210 (2016).

[46] D.A. Bies, C.H. Hansen, Engineering Noise Control: Theory and Practice (Spon Press Fourth ed., Abingdon, UK, 2009, pp. 737) 